\title{
La structure des liquides simples et des alliages liquides métalliques
}

\author{
V. Simonet et R. Bellissent ${ }^{1}$ \\ Laboratoire Louis Néel, UPR 5051 du CNRS, BP. 166, 38042 Grenoble cedex 9, France \\ ${ }^{1}$ DRFMC/SPSM-MDN, CEA-Grenoble, 17 rue des Martyrs, 38402 Grenoble cedex, France
}

\begin{abstract}
Résumé : De par le caractère isotrope des interactions et leur courte portée, les liquides métalliques ont longtemps été considérés comme des systèmes modèles pouvant être décrits par un arrangement aléatoire de sphères dures. Ceci a été confirmé par l'allure des premières fonctions de distribution de paires déterminées par diffusion de $\mathrm{RX}$ ou de neutrons à la précision près des expériences de l'époque. Néanmoins, cette image a tout d'abord été révisée pour le cas des alliages métalliques dont les fonctions de distribution de paires ont révélé un ordre local plus complexe à cause des affinités chimiques entre espèces différentes. Grâce à la possibilité actuelle de mesurer des facteurs de structure de bien meilleure qualité, la question de la nature de l'ordre local et du degré d'isotropie des interactions peut se poser même dans le cas des liquides simples ou dans le cas d'alliages présentant à priori peu d'ordre chimique. Dans ce cadre, l'ordre local icosaédrique est particulièrement intéressant puisque l'icosaèdre est un agrégat très compact et, bien que présentant des liaisons directionnelles, très proche d'une configuration isotrope. La mise en évidence de ce type d'ordre local est présentée pour deux types de liquides : les liquides surfondus dans lesquels un ordre local icosaédrique a été prédit pour expliquer les propriétés de surfusion, et les liquides en équilibre avec des quasicristaux qui présentent un ordre local icosaédrique.
\end{abstract}

\section{Introduction aux liquides métalliques}

\subsection{Liquides monoatomiques}

Les éléments métalliques dans l'état liquide représentent une part importante du tableau périodique des éléments (cf. Fig. 1). Ils sont caractérisés par la coexistence d'électrons de conduction délocalisés et d'un arrangement dynamique d'ions positifs et présentent une bonne conductivité thermique et électrique. Ils sont notablement différents des liquides moléculaires ou semiconducteurs (caractérisés par des liaisons de nature covalente) de par leur plus grande compacité (nombre de coordination entre 9 et 12) et le caractère isotrope des interactions atomiques en première approximation. Notons que certains éléments, tels que le Ge ou le Te, subissent une transition semi-conducteur/métal dans l'état liquide.

Le problème de la détermination de la structure de l'état liquide est difficile car c'est un problème à $\mathrm{N}$ particules en interaction. Dans le cas de l'état cristallin, cette difficulté est surmontée par la périodicité de translation de l'édifice atomique qui détermine les positions atomiques à partir d'un nombre réduit de paramètres. Dans le cas du gaz, l'absence d'interaction entre des atomes ponctuels situés à de grandes distances permet un traitement de physique statistique. Le liquide peut sembler proche de l'état solide car il a une densité comparable dans des conditions voisines de température et de pression. En outre, sa chaleur latente de fusion est plus petite que la chaleur latente de vaporisation, ce qui implique que les deux états, solide et liquide, ont des propriétés de liaison voisines. D'un autre côté, la similarité des deux états liquide et gaz 


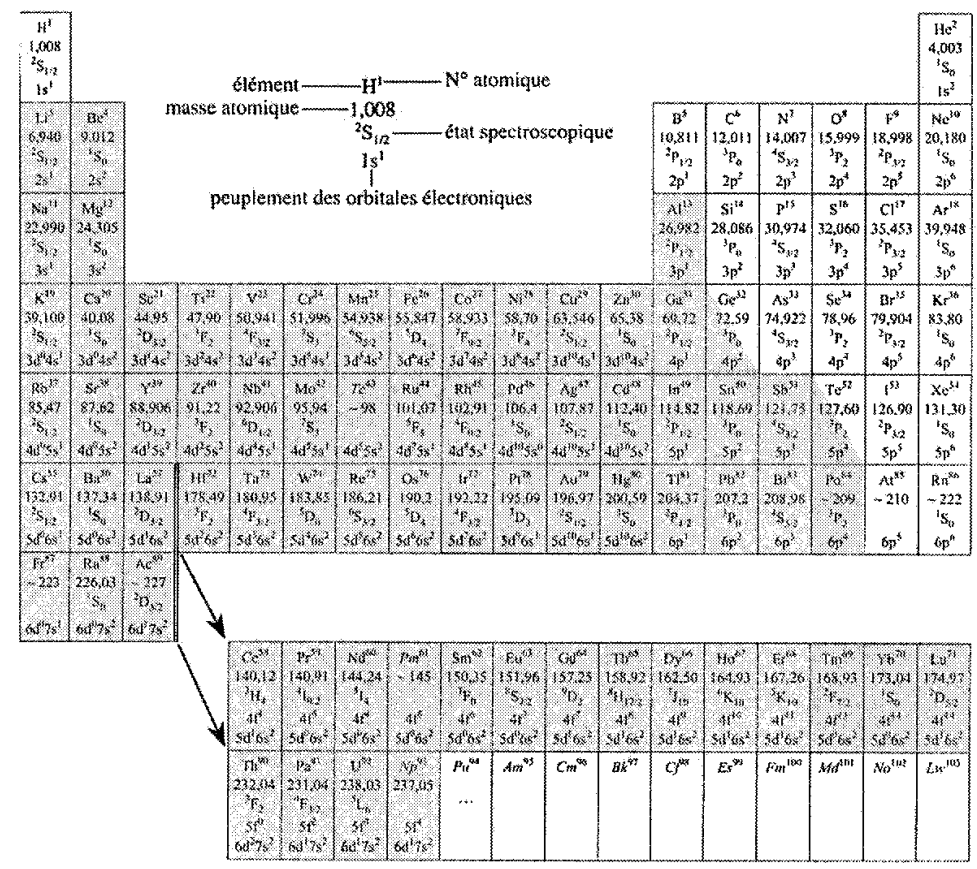

Figure 1 : Les éléments en grisé dans ce tableau périodique correspondent à des liquides métalliques.

est indiquée par le fait qu'il est possible de passer continûment de l'un à l'autre en contournant le point critique. Cependant, les liquides different de l'état solide et du gaz par l'absence d'ordre à grande portée d'une part et par la présence d'interactions entre les atomes qui induisent un ordre à courte portée et un caractère non-aléatoire des positions atomiques d'autre part. De ce fait, les méthodes appliquées pour décrire la structure des gaz ou celle des cristaux ne sont plus valables dans le cas des liquides.

Des informations sur les propriétés structurales et thermodynamiques d'équilibre d'un liquide peuvent être obtenues grâce à la connaissance de la fonction de distribution de paire $g(r)$. Celle-ci correspond à la probabilité de trouver un autre atome à une distance $r$ de l'atome à l'origine. Elle est déterminée à partir du facteur de structure $S(Q)$ obtenu lors d'expériences de diffraction, avec $Q$ le transfert de moment :

$$
\begin{gathered}
Q(S(Q)-1)=4 \pi \rho \int r(g(r)-1) \sin (Q r) d r \\
r(g(r)-1)=\frac{1}{2 \pi^{2} \rho} \int Q(S(Q)-1) \sin (Q r) d Q
\end{gathered}
$$

où $\rho$ est la densité moyenne du système diffuseur.

La représentation schématique du spectre de diffraction d'un cristal, d'un liquide et d'un gaz est montrée sur la figure 2. Le spectre de diffraction d'un cristal est constitué d'un arrangement de pics de Bragg. Le facteur de structure d'un gaz idéal ne présente à l'inverse pas de maxima 

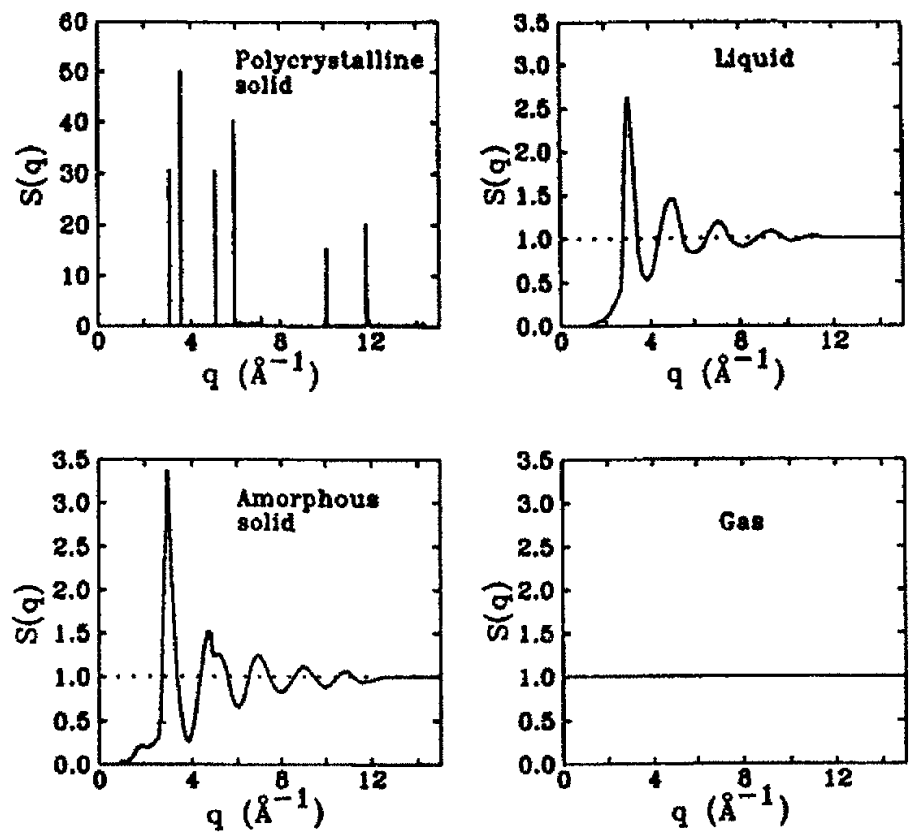

Figure 2 : Facteurs de structure schématiques de matériaux dans l'état solide, cristallin ou amorphe, liquide, et gaz.

mais une diffusion continue. ${ }^{1}$ La première détermination du facteur de structure d'un liquide par diffraction de rayons X fut réalisée par Debye et Menke dans du mercure en 1929 [1] et montra la présence, dans le facteur de structure, d'oscillations interprétées comme des fluctuations dans la distribution atomique. La technique de diffraction de RX fut la seule utilisée pour mesurer le facteur de structure des liquides jusque dans les années 60 . A partir de ces années correspondant à la construction des premiers réacteurs de neutrons, la diffusion neutronique se révéla un excellent outil pour l'étude de la structure des liquides. Notons toutefois que la connaissance des fonctions de distribution de paires est insuffisante pour déterminer entièrement la structure des liquides car il n'y a pas d'information sur les corrélations entre les positions de plus de deux particules et donc en particulier sur la dépendance angulaire de l'arrangement des voisins.

Il est également important de souligner que la grande majorité des expériences de structure des liquides est réalisée sur des spectromètres deux-axes sans analyse en énergie. Dans le cas de la diffusion de neutrons, un faisceau monochromatique est diffusé par le liquide et les neutrons émis sont détectés en fonction de l'angle $2 \theta$ du multidétecteur. Cette technique équivaut à intégrer sur l'énergie finale des neutrons. Dans un liquide où les atomes sont en mouvement, il n'y a pas de diffusion élastique. On peut néanmoins se placer dans le cadre de l'approximation statique (norme égale des vecteurs d'onde des neutrons incidents et diffusés) en considérant que les transferts d'énergie entre les neutrons et le système diffusant sont petits par rapport à l'énergie des neutrons incidents.

\footnotetext{
${ }^{1}$ En fait, le facteur de structure d'un gaz réel a un maximum faible et une remontée très importante à petits vecteurs de diffusion due aux fortes valeurs de compressibilité.
} 


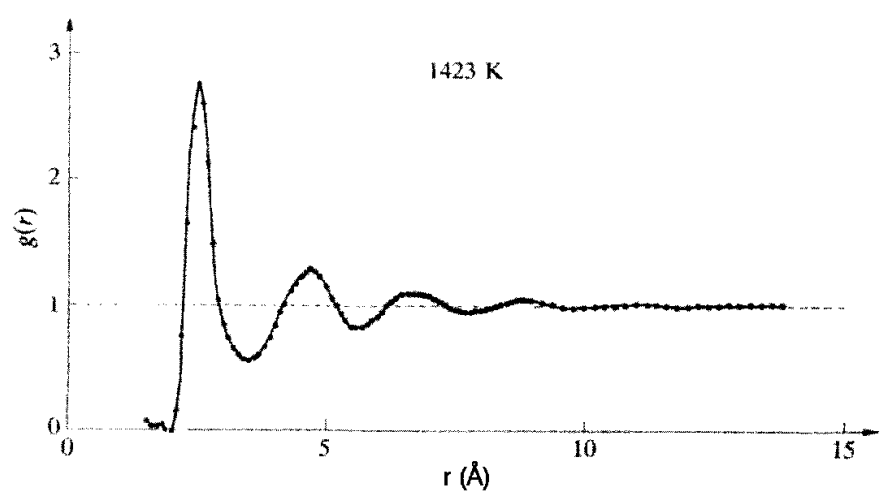

Figure 3 : Fonction de distribution de paires $g(r)$ du cuivre liquide près du point de fusion [3].

$$
S(Q)=\int S(Q, \omega) d \omega=\int G(r, t) \exp (i Q r) \exp (-i \omega t) d r d t d \omega=\int G(r, 0) \exp (i Q r) d r
$$

avec la fonction de corrélation de paires $G(r, 0)=\delta(r)+\rho g(r)$ où $\delta(r)$ résulte de l'autocorrélation. Ainsi, la section efficace différentielle est reliée à la corrélation entre les positions de deux atomes au même instant. Sa détermination équivaut donc à réaliser une photo instantannée du système. En pratique, on corrige les spectres mesurés des écarts à l'approximation statique en appliquant la correction de Placzek [2].

Avant de discuter la nature de l'information structurale qui est accessible via les fonctions de distribution de paires, notons que le facteur de structure peut être directement relié à des grandeurs physiques. Les conditions aux limites du facteur de structure sont 1 aux grandes valeurs de $Q$ et $S(0)=k_{B} T \rho \kappa_{T}$ avec $\kappa_{T}$ la compressibilité isotherme du liquide et $\rho$ la densité moyenne. La valeur non-nulle du facteur de structure à $Q=0$ fournit une estimation des fluctuations de densité du liquide ce qui constitue une autre approche de l'ordre du système ${ }^{2}$. Enfin, les propriétés de transport électronique peuvent être estimées à partir du facteur de structure grâce à une approche théorique de Ziman basée sur un modèle d'électrons presque libres [4].

Les fonctions de distribution de paires déduites des expériences de diffraction pour l'ensemble des liquides ont la même allure générale (cf. Fig. 3). $g(r)$ est nulle en dessous d'une certaine distance correspondant au rayon effectif du cour dur des atomes. La présence d'un premier minimum dans le potentiel d'interaction se traduit par une première couche de coordination qui entraîne un pic intense à une distance correspondant aux premières distances interatomiques. Il est suivi d'un deuxième minimum dî au non-recouvrement des couches atomiques et ainsi de suite avec des oscillations successives qui s'amortissent pour les distances inter-atomiques croissantes $(g(r)$ tend vers 1 pour $r$ tendant vers l'infini). Ceci traduit l'atténuation des corrélations de positions entre atomes à grande distance. La spécificité des liquides métalliques est une décroissance à peu près uniforme des oscillations. Ces oscillations révèlent un ordre local s'étendant typiquement sur 4-5 couches atomiques au dessus du point de fusion. La nature de cet ordre local est géré par un potentiel d'interaction atomique à peu près isotrope et à décroissance rapide. Du point de vue quantitatif, les fonctions de distribution de paires permettent d'évaluer des paramètres structuraux tels que les distances moyennes inter-atomiques et les nombres de coordination. Ces

\footnotetext{
${ }^{2}$ L'étude des fluctuations de densité fait l'objet d'un grand nombre d'expériences aux petits angles.
} 

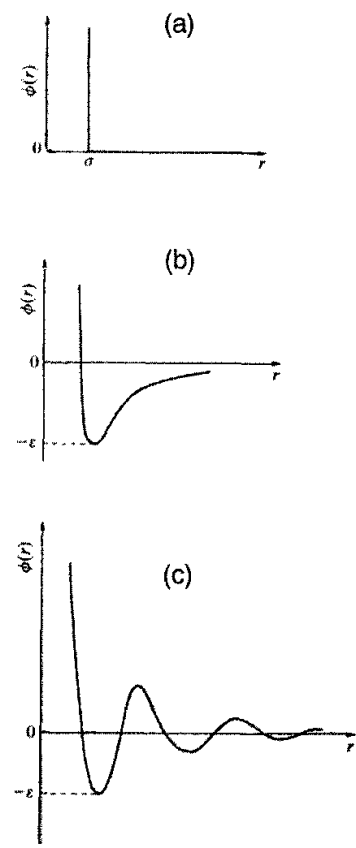

Figure 4 : Différents modèles de potentiels d'interaction de paires : (a) sphères dures, (b) Lennard-Jones et (c) potentiel oscillant.

derniers ne sont néanmoins généralement connus qu'avec une précision de $10 \%$ en valeur absolue, la précision sur la variation relative du nombre de voisins étant bien meilleure. Ils sont obtenus via la fonction de distribution radiale $\left(R D F(r)=4 \pi \rho r^{2} g(r)\right)$ définie comme la densité de probabilité, un atome étant à l'origine d'en trouver un à la distance $r$ toutes directions confondues. Dans les liquides métalliques, les couches atomiques ne sont généralement pas bien séparées et la détermination de l'aire caractéristique d'une couche atomique est arbitraire. Enfin, notons que, en toute rigueur, la notion de nombre de coordination n'a de sens que dans un solide où les distances interatomiques sont quantifiées. Dans le cas d'un liquide, la fonction de distribution de paires permet seulement de déterminer un nombre moyen de voisins sur une couche sphérique.

Plusieurs modèles ont été élaborés pour rendre compte des fonctions de distribution de paires à partir de différents potentiels d'interaction $\phi(r)$ (cf. Fig. 4). Le plus simple est le potentiel de sphères dures qui ne comprend qu'une partie répulsive : $\phi(r)=\infty$ pour $r<r_{0}$ et $\phi(r)=0$ pour $r>r_{0}$. Citons également le potentiel de Lennard-Jones plus mou avec une partie répulsive et une partie attractive : $\phi(r)=4 \epsilon\left\{\left(\frac{r}{r_{0}}\right)^{12}-\left(\frac{r}{r_{0}}\right)^{6}\right\}$. Dans les liquides métalliques, le potentiel effectif ion-ion doit présenter des oscillations amorties de Friedel dues à la présence d'électrons de conduction. Des équations, permettant de relier ces potentiels d'interaction de paires aux fonctions de distribution de paires, ont été développées moyennant certaines approximations. Celles-ci consistent essentiellement à exprimer les fonctions de distribution ou potentiel d'interaction à plus de 2 corps par des combinaisons de fonctions à 2 corps. Parmi ces méthodes, citons les équations de Yvon-Born-Green, de chaîne hypertressée ou de Percus-Yevick. L'expression de cette dernière s'écrit pour un liquide monoatomique : 


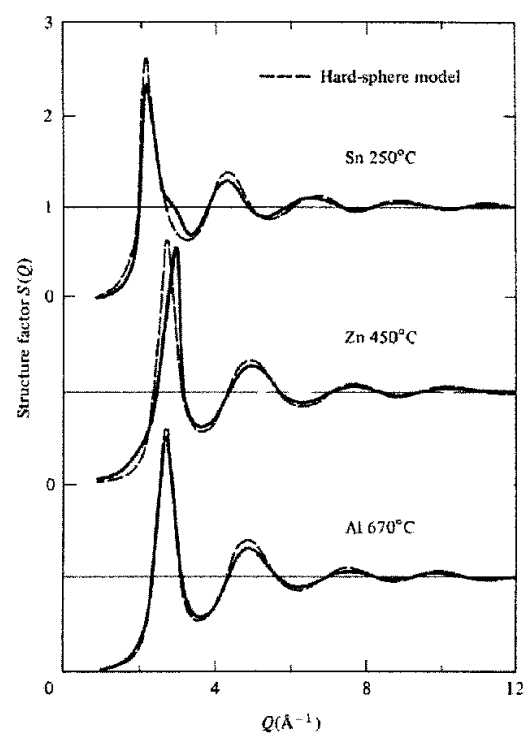

Figure 5 : Facteur de structure des liquides $\mathrm{Sn}, \mathrm{Zn}$ et $\mathrm{Al}$ et leur simulation de sphères dures basée sur l'équation de Percus-Yevik [5].

$$
g(r) \exp \left(\frac{\phi(r)}{k_{B} T}\right)=1+\rho \int\left(g\left(r^{\prime}\right)-1\right)\left(1-\exp \left(\frac{\left|r-r^{\prime}\right|}{k_{B} T}\right)\right) g\left(\left|r-r^{\prime}\right|\right) d r^{\prime}
$$

Le succès de cette équation intégrale provient de sa grande simplicité. La justification de sa validité est basée sur le fait que les termes négligés, à plus de deux atomes, se compensent de fait partiellement. Dans les autres approches, les termes d'ordre supérieurs en nombre de voisins forment des séries alterées à convergence très lente. L'équation de Percus-Yevick conduit en outre à une solution analytique avec un potentiel de sphères dures. Les facteurs de structure peuvent alors être simulés à partir de deux paramètres ajustables que sont le diamètre des sphères dures $\sigma$ et la compacité ou taux d'empilement $\eta=\pi \rho \sigma^{3} / 6$.

Pour achever le panorama de l'approche théorique de la structure des liquides, citons les méthodes de simulations numériques, également largement employées pour évaluer la structure des systèmes désordonnés. C'est le cas des méthodes de Monte Carlo qui consistent à générer aléatoirement un grand nombre de configurations atomiques dont la probabilité de chacune est calculée à partir du potentiel d'interaction de paires et du facteur de Boltzmann. Citons également la méthode de dynamique moléculaire où, à partir d'une configuration initiale, les atomes accomplissent une série de petits déplacements calculés en résolvant à chaque étape le hamiltonien classique du système pour un potentiel de paire donné, avant de recommencer l'opération. C'est la méthode la plus rigoureuse pour tester un potentiel de paire car sa précision n'est limitée que par la taille du système et la portée que l'on donne aux interactions.

Malgré l'augmentation de la complexité des potentiels d'interaction ou des algorithmes de calcul utilisés pour décrire la structure des liquides, un résultat frappant est que l'allure générale des courbes expérimentales est largement déterminée par la partie répulsive du potentiel. En effet, un simple modèle de Percus-Yevick basé sur un potentiel de sphères dures (avec une compacité de 0.45 ) rend compte, en première approximation, des fonctions de distribution de paires, du moins 


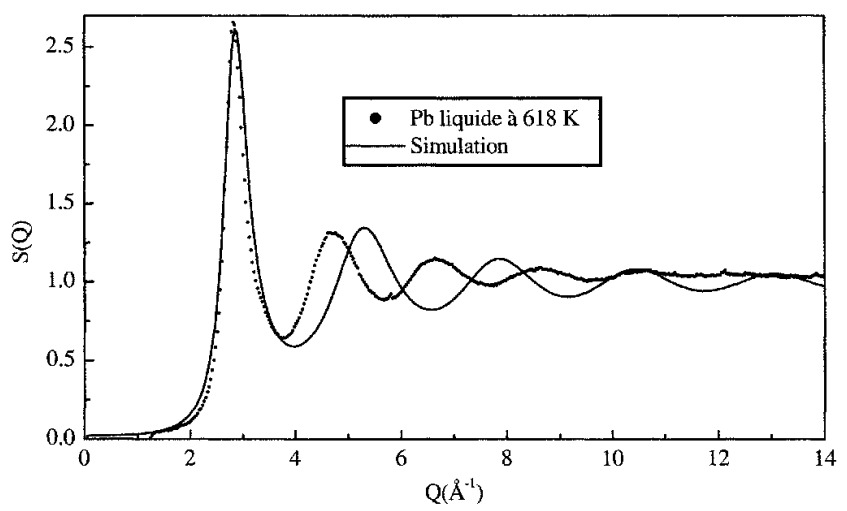

Figure 6 : Comparaison du facteur de structure mesuré du $\mathrm{Pb}$ liquide à $618 \mathrm{~K}$ et d'une simulation basée sur l'équation de Percus-Yevik avec des sphères dures de rayon 2.4 $\AA$ et une compacité de 0.47.

de celles mesurées dans les années 70 . Ceci est illustré sur la figure 5 pour trois liquides $\mathrm{Al}$, Sn et Zn $[5,6]$. Certains écarts à la simulation sont néanmoins déja observables tels qu'une asymétrie du premier pic pour le $\mathrm{Zn}$ ou un épaulement sur la droite de la première oscillation pour le Sn. Ces écarts caractérisent la nature légèrement directionnelle des liaisons persistant dans le liquide. Il a fallu attendre l'amélioration des sources de neutrons et l'avènement des sources de rayonnement synchrotron pour que des mesures de facteurs de structure de grande qualité permettent de mettre clairement en évidence et d'analyser ce type de déviations par rapport à l'image d'un liquide de sphères dures. Un exemple révélateur est celui du plomb liquide. Contrairement à ce qui était admis, son facteur de structure, récemment remesuré, ne peut être correctement décrit dans l'approximation de Percus-Yevick par un modèle de sphères dures (cf. Fig. 6).

La dépendance en température des paramètres structuraux extraits des fonctions de distributions de paires met également en évidence l'importance de la partie attractive du potentiel inter-atomique. En effet, l'expansion thermique ne se traduit pas simplement par une augmentation isotrope des distances inter-atomiques mais par un réarrangement de l'ordre local avec des distances premiers-voisins qui restent à peu près constantes et une diminution du nombre de coordination [7]. Notons que l'étude de l'évolution de la structure et des propriétés électroniques des liquides en fonction de la température (et de la pression) dans un domaine proche du domaine supercritique ou dans le domaine surfondu est, à l'heure actuelle, un des axes de recherche les plus explorés.

\section{$1.2 \quad$ Alliages liquides}

Dans le cas des alliages liquides métalliques, la complexité de la structure et des propriétés augmente drastiquement même pour le cas le plus simple d'alliages binaires. Les affinités entre différentes espèces chimiques doivent être prises en considération car elles produisent une structure généralement plus complexe qu'un mélange aléatoire des différentes espèces chimiques. En particulier, on observe souvent l'apparition d'un ordre chimique à courte portée plus ou moins marqué (les espèces chimiques différentes s'attirent) ou, à l'inverse, une tendance à la démixtion (les espèces chimiques identiques s'agrègent). Du point de vue expérimental également, les études sont beaucoup plus lourdes. Dans le cas d'un alliage binaire en effet, la connaissance de trois fonctions de distributions de paires partielles est nécessaire pour décrire la structure (soient $\mathrm{N}(\mathrm{N}+1) / 2$ 
pour un alliage à $\mathrm{N}$ composantes).

Les outils d'analyse de ces structures reposent donc sur la détermination des facteurs de structure et fonctions de distribution de paires partiels. Pour un alliage binaire, ceux-ci sont accessibles via la mesure de trois facteurs de structure en faisant varier le pouvoir diffusant d'un des composants de l'alliage par substitution isotopique (en substituant un élément par ses isotopes) ou isomorphe (en substituant aléatoirement un élément par un élément voisin) pour des expériences de diffusion de neutrons, ou en utilisant la diffusion anomale en rayons $\mathrm{X}$ (en théorie également possible en neutrons), soit enfin en combinant les facteurs de structure déterminés par les différents types de mesure. La décomposition des facteurs de structure totaux en trois composantes partielles pour des alliages binaires peut s'effectuer suivant différents formalismes, en particulier ceux de Faber-Ziman et Bhatia-Thornton ${ }^{3}$. Considérons le cas de la diffusion de neutrons.

Dans le formalisme de Faber-Ziman [9] pour un alliage binaire composé d'atomes $A$ et $B$, le facteur de structure de Faber-Ziman $I_{F Z}$, défini à partir de la section efficace différentielle, se décompose en une combinaison linéaire des facteurs de structure partiels $S_{i j}$ avec $i, j=A, B$ :

$$
\begin{aligned}
& \left.I_{F Z}(Q)=\frac{1}{<b\rangle^{2}}\left(\frac{d \sigma}{d \Omega}(Q)-\frac{\sigma_{i n c}}{4 \pi}-\left(<b^{2}\right\rangle-\langle b\rangle^{2}\right)\right) \\
= & \frac{c_{A}^{2} b_{A}^{2}}{\langle b\rangle^{2}} S_{A A}(Q)+\frac{c_{B}^{2} b_{B}^{2}}{\langle b\rangle^{2}} S_{B B}(Q)+\frac{2 c_{A} c_{B} b_{A} b_{B}}{<b\rangle^{2}} S_{A B}(Q)
\end{aligned}
$$

avec $\sigma_{i n c}=\sum_{j} c_{j} \sigma_{i n c}^{j}$ la section efficace de diffusion incohérente intrinsèque de l'alliage, $<$ $\left.b^{2}\right\rangle=\sum_{j} b_{j}^{2}$ et $\langle b\rangle=\sum_{j} b_{j}$ où $b_{j}$ est la longueur de diffusion cohérente de l'élément $j$. On accède aux probabilités, un atome de type $A$ (resp. $B$ ) étant à l'origine, d'en trouver un de type $A$ ou $B$ à la distance $r$ grâce aux fonctions de distribution de paires partielles $g_{i j}$ :

$$
r\left(g_{i j}(r)-1\right)=\frac{1}{2 \pi^{2} \rho} \int Q\left(S_{i j}(Q)-1\right) \sin (Q r) d Q
$$

Un autre formalisme est couramment employé qui a été développé par Bhatia et Thornton [10] pour mieux mettre en évidence les corrélations chimiques entre espèces au sein de l'alliage. Le facteur de structure de Bhatia-Thornton $S_{B T}$ s'écrit en fonction des facteurs de structure partiels $S_{i j}$ avec $i, j=N, C$.

$$
\begin{gathered}
S_{B T}(Q)=\frac{1}{\left.<b^{2}\right\rangle}\left(\frac{d \sigma}{d \Omega}(Q)-\frac{\sigma_{i n c}}{4 \pi}\right) \\
=\frac{\langle b\rangle^{2}}{\left.<b^{2}\right\rangle} S_{N N}(Q)+\frac{2 \Delta b<b>}{\left.<b^{2}\right\rangle} S_{N C}(Q)+\frac{c_{A} c_{B}(\Delta b)^{2}}{\left.<b^{2}\right\rangle} S_{C C}(Q)
\end{gathered}
$$

avec $\Delta b=b_{A}-b_{B}$

On détermine par transformée de Fourier (Eq. (6)) les fonctions $g_{i j}(r)$ qui renseignent sur les corrélations entre la densité atomique totale de l'alliage $(N)$ et l'écart à la concentration moyenne $(C)$.

$S_{N N}$ rend compte de l'ordre topologique. C'est la diffusion cohérente d'un système où tous les atomes ont la même longueur de diffusion, à savoir la longueur de diffusion moyenne de l'alliage. Notons qu'il présente une remontée caractéristique à petits $Q$ pour des alliages liquides présentant des propriétés de démixtion à cause de la présence de domaines de taille importante par rapport au volume atomique moyen, diffusant de manière différente.

\footnotetext{
${ }^{3}$ Un autre formalisme est utilisé, celui de Ashcroft et Langreth, qui fournit des informations similaires à celui de Faber-Ziman [8].
} 


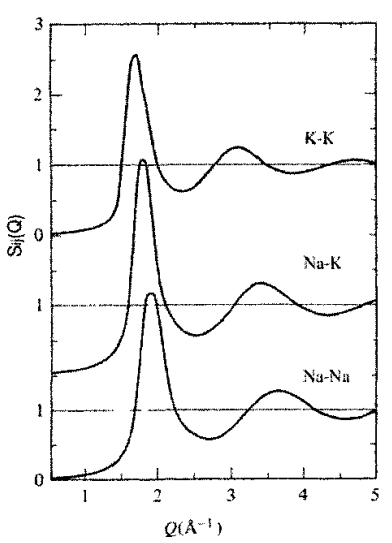

(a)

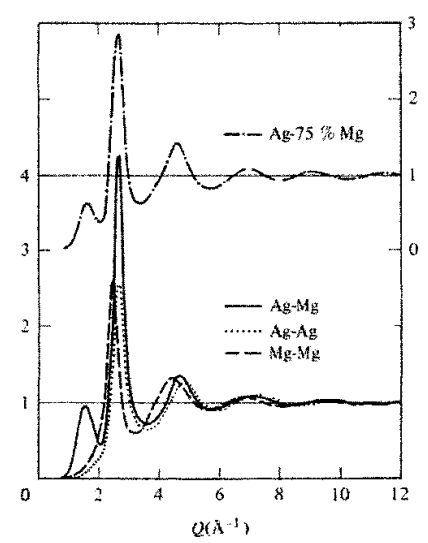

(b)

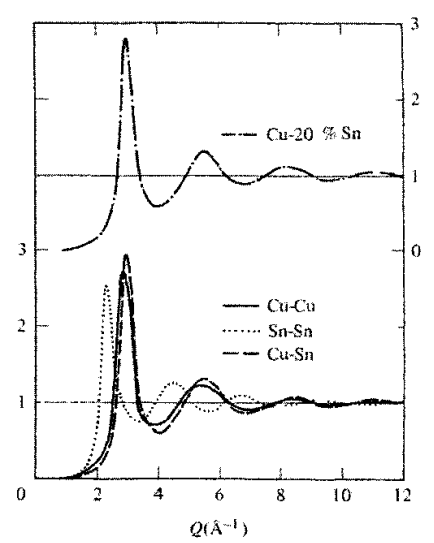

(c)

Figure 7 : Exemple de facteurs de structure partiels d'alliages liquides métalliques dans le formalisme d'Ashcroft-Langreth avec l'hypothèse d'indépendance en fonction de la composition.

$S_{C C}$ est une mesure des fluctuations de concentration et rend donc compte de l'ordre chimique au sein de l'alliage. L'amplitude et le signe des oscillations de $g_{C C}$ indiquent la préférence pour des atomes voisins de même nature (signe + ) ou de nature différente (signe -). $g_{C C}$ oscille autour de 0 et intègre deux effets : un effet de taille et un effet chimique. Si les atomes ont des tailles différentes, une oscillation de $g_{C C}$ apparait au voisinage du maximum de $g_{N N}$. S'il y a tendance à l'ordre chimique (resp. à la ségrégation), $g_{C C}$ devient négatif (positif) au voisinage du premier maximum de $g_{N N}$ et positif (négatif) au voisinage du second maximum.

$S_{N C}$ a une signification moins claire que les précédents. Il rend compte du fait que, lorsque les atomes $\mathrm{A}$ et $\mathrm{B}$ n'ont pas la même taille, des variations d'ordre chimique entrainent des évolutions de la densité locale. Ce terme s'annule pour des atomes de même taille.

Dans de nombreux cas, un prépic est observé sur le facteur de structure total à une position en $Q$ inférieure à la première oscillation intense. Ce prépic est souvent dû à l'alternance d'éléments de longueurs de diffusion différentes et est donc caractéristique de l'ordre chimique (apparait sur $S_{C C}$ ). Des effets de taille peuvent s'ajouter, à savoir que si les atomes de petites tailles sont les plus diffusants, l'effet d'alternance est renforcé et le prépic est plus intense $\left(S_{C C}\right.$ renforcé par $S_{N C}$ ). A l'inverse, si les atomes de petite taille sont les moins diffusants, l'effet est atténué, voire annulé ( $S_{C C}$ et $S_{N C}$ se compensent). Un autre intérêt du formalisme de Bhatia-Thornton est qu'il permet de faire apparaître de façon simple la relation entre les différents facteurs de structure et les quantités thermodynamiques telles que la compressibilité isotherme $\kappa_{T}$ ou l'énergie libre molaire $G$. Ainsi, dans la limite des vecteurs de diffusion nuls, les facteurs de structure partiels s'écrivent :

$$
\begin{gathered}
S_{N N}(0)=\rho k_{B} T \kappa_{T}+\delta^{2} c_{A} c_{B} S_{C C}(0) \\
S_{C N}(0)=-c_{A} c_{B} \delta S_{C C}(0) \\
S_{C C}(0)=N_{a} k_{B} T /\left[c_{A} c_{B}\left(\frac{\partial^{2} G}{\partial c_{A}^{2}}\right)_{T, P}\right]
\end{gathered}
$$


$N_{a}$ est le nombre d'Avogadro et $\delta=\left(\nu_{A}-\nu_{B}\right) /\left(c_{A} \nu_{A}+c_{B} \nu_{B}\right)$ le facteur de dilatation avec $\nu_{A}$ et $\nu_{B}$ les volumes molaires partiels des deux espèces chimiques. Cette procédure a été généralisée pour des alliages ternaires [11] ou à $\mathrm{N}$ composantes [12].

La procédure délicate de mesure de trois facteurs de structure et d'extraction des fonctions de distribution de paires partielles dans les liquides métalliques binaires n'a été menée que dans assez peu de cas. Citons par exemple la première expérience de diffusion de neutrons utilisant la substitution isotopique dans le liquide CuSn [13] ou encore les expériences de Waseda et coll. basées sur la diffusion anomale des RX (CeNi [14]). Face à la difficulté de ces expériences, un certain nombre d'auteurs ont fait l'hypothèse de l'indépendance des facteurs de structure partiels avec la composition. Une étude en fonction de la concentration du liquide serait alors suffisante pour obtenir les fonctions de distribution de paires partielles. Des exemples typiques des fonctions ainsi obtenues sont montrés sur la figure 7 avec le cas d'un liquide desordonné en (a), d'un liquide avec de l'ordre chimique présentant un prépic en (b) ou encore d'un liquide désordonné mais avec effet de taille (rayon atomique des espèces chimiques diffèrent) en (c). L'hypothèse d'indépendance en fonction de la concentration n'est cependant strictement valable que pour des liquides avec des espèces chimiques de même rayon atomique et sans ordre chimique, ce qui est extrêmement restrictif. Dans certains cas, les partiels déterminés selon cette hypothèse se sont avérés très différents de ceux mesurés par la suite dans les mêmes composés en utilisant la technique correcte de substitution isotopique.

La complexité de l'ordre local dans les alliages liquides a longtemps été décrite sur une base purement chimique, le liquide étant encore considéré comme constitué d'une assemblée de sphères dures. Ainsi, Blétry [15] a calculé les évolutions des facteurs de structure partiels à partir d'un assemblage compact de sphères dures (au sein duquel des lacunes sont aléatoirement distribuées) et en imposant un désordre ou un ordre chimique. Il a ainsi pu expliquer qualitativement l'allure des facteurs de structure partiels expérimentaux d'alliages liquides désordonnés (CuSn [13]), avec ordre chimique ( $\mathrm{CoP}$ [16], $\mathrm{LiPb}, \mathrm{LiAg}$ [17]) ou tendance à la démixtion ( $\mathrm{CuPb}$ [18]). Les facteurs de structure partiels de ces derniers liquides, obtenus dans le formalisme de Bhatia-Thornton sont montrés sur la figure 8 .

Un premier pas vers un ordre topologique différent du modèle de sphères dures a été franchi pour décrire l'ordre local des alliages liquides formés d'un métal alcalin et d'un élément du groupe post-métal de transition (exemple : $\mathrm{CsSb}, \mathrm{LiPb}, \mathrm{NaSn}$ etc.). Ces liquides, bien que formés de deux éléments dont le liquide pur est métallique, présentent pour certaines compositions des transitions électroniques avec une chute de la conductivité électrique. Pour ces mêmes compositions, leur facteur de structure total a une allure singulière avec en particulier un prépic intense qui ne peut être uniquement relié à $S_{C C}$ mais est aussi présent sur $S_{N N}$. Ceci peut être expliqué par la formation de complexes dans le liquide, similaires à ceux des phases solides, dû à un transfert de charge entre éléments chimiques aboutissant à des liaisons de nature ionique ou covalente $[19,20,21]$.

Cependant, dans les liquides métalliques (mono- ou poly-atomiques) plus standards, l'ordre topologique n'a pas été examiné en détail à cause de la nature supposée isotrope des interactions inter-atomiques et de la difficulté de sa mise en évidence expérimentale. Dans la suite, nous allons illustrer l'intérêt que présente la détermination de l'ordre local topologique, au-delà du modèle de sphères dures, avec l'exemple de la recherche de l'ordre local icosaédrique.

\subsection{Ordre local icosaédrique}

Il est intéressant de faire un rapprochement entre l'étude de l'ordre local des liquides métalliques et celui des amorphes métalliques, souvent décrits comme des liquides gelés. Ces phases metastables ont des facteurs de structure assez proches de ceux des liquides à l'équilibre thermodyna- 

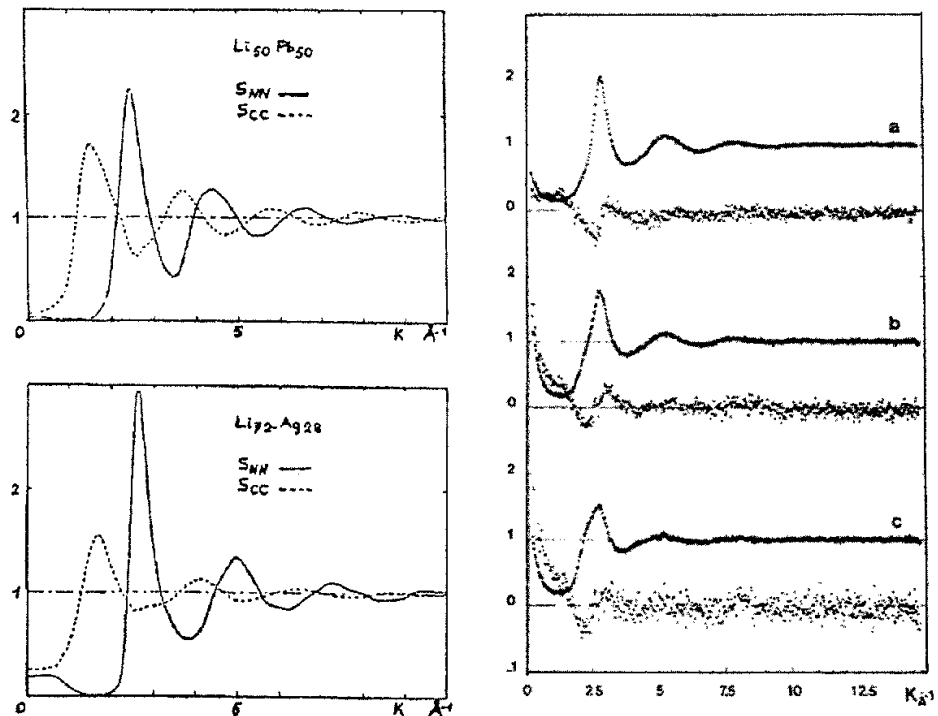

Figure 8 : Facteurs de structure partiels dans le formalisme de Bhatia-Thornton d'alliages liquides métalliques. Figure de gauche : $S_{N N}$ et $S_{C C}$ pour les liquides LiPb et LiAg avec ordre chimique [17]. Figure de droite : $S_{N N}$ et $S_{N C}$ pour les liquides $\mathrm{CuPb}$ avec 81 (a), 64.5 (b) et 49 (c) \% Cu présentant une tendance à la démixtion [18].

mique. Des efforts importants ont été entrepris pour comprendre la nature de l'ordre topologique dans ces systèmes. Le facteur de structure mesuré des amorphes métalliques est constitué d'un premier pic étroit suivi d'une deuxième oscillation dédoublée et d'un amortissement des oscillations à plus grands $Q$ (cf. Figs. 2 et 9). Le nombre de coordination est compris entre 11 et 12 . C'est le cas pour les amorphes métalliques monoatomiques tels que le $\mathrm{Co}$ [22], mais également pour la majorité des alliages métal-métal ou métal-métallö̈de, tels que MgZn [23] ou FeB [24].

Des tentatives de modélisation à partir d'empilements compacts de sphères dures ont été réalisées "à la main" (modèle de Bernal [25]) ou à partir de différents algorithmes (modèle de Bennett [26]) dont certains favorisent l'ordre local tétraédrique comme c'est le cas des modèles de Ichikawa [27], Blétry [28] et Sadoc-Dixmier [29]. Dans ce dernier cas, l'empilement des tétraèdres réguliers autour de centres forme des icosaèdres parfaits. Ce modèle n'est néanmoins pas très compact (compacité de 0.51 comparée à la valeur expérimentale de 0.69). Ceci est dû tout d'abord au fait que ce modèle, comme les autres modèles statiques, doit être relaxé. D'autre part, la compacité peut être augmentée en choisissant un mélange de sphères de tailles légèrement différentes. En effet, on peut placer les plus petites sphères au centre des icosaèdres afin de prendre en compte la différence entre la distance du centre à la surface de l'icosaèdre et la distance de deux sphères sur la surface de l'icosaèdre. Ainsi, avec $15 \%$ de sphères de taille $10 \%$ inférieure, le facteur de structure calculé présente un dédoublement de la seconde oscillation et une compacité supérieure. Enfin, Sachdev et Nelson [30] ont montré dans le cadre de la théorie de Landau, en partant d'un réseau icosaédrique construit sur la surface d'une sphère à 4 dimensions, que la symétrie icosaédrique induit des maxima à des positions caractéristiques dans le facteur de structure des verres métalliques. L'application de cette théorie au cas du Co amorphe est illustrée sur la figure 9 . 


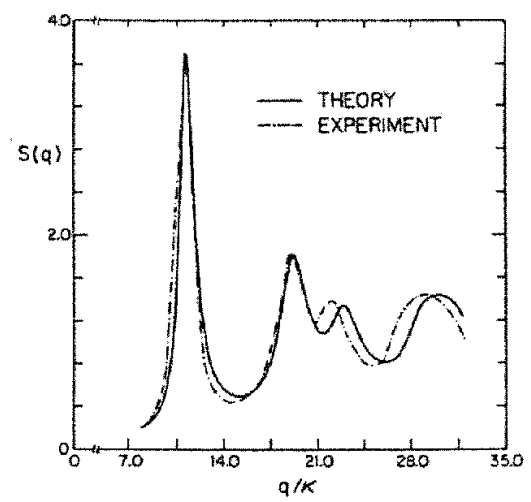

Figure 9 : Comparaison des facteurs de structure du Co amorphe mesuré [22] et calculé d'après la. théorie de Sachdev et Nelson [30].

On peut à présent se demander ce qu'il en est des liquides à l'équilibre et également dans l'état surfondu, c'est-à-dire maintenu liquide à une temperature inférieure à la température de fusion du solide. En 1952, Frank proposa une explication des propriétés de surfusion de liquides métalliques simples [31], mises en évidence par Turnbull [32], basée sur la présence préférentielle de l'agrégat icosaédrique dans ces liquides surfondus. L'icosaèdre, agrégat compact de 13 atomes, possède 6 axes de rotation d'ordre 5 (cf. Fig. 10). Cet agrégat est plus symétrique que les deux autres arrangements compacts de 13 atomes, à savoir hexagonal compact (hc) et cubique faces centrées (cfc), mais contrairement à ces derniers, n'est pas compatible avec la périodicité de translation. D'autre part, l'énergie de liaison d'un tel agrégat, calculée avec un potentiel de Lennard-Jones, est $8.4 \%$ plus basse que pour les deux autres agrégats. Récemment, D. Holland-Moritz et coll. ont réalisé des expériences de surfusion par une technique de lévitation électromagnétique sur différents types de liquides [33]. L'analyse des profils de température a permis de relier le degré de surfusion à l'ordre local du solide formé. Le degré de surfusion décroît du solide de structure cubique centré (cc), à cfc, puis à des phases cristallines plus complexes (polytétrahédrales) et enfin à des quasicristaux dont l'ordre local est icosaédrique. Selon l'hypothèse d'un ordre local icosaédrique dans les liquides surfondus, cette évolution peut s'expliquer qualitativement par l'augmentation de l'énergie d'interface entre liquide et germe solide avec la différence d'ordre local liquide/solide.

Du point de vue expérimental, peu de résultats concernant l'ordre local icosaédrique dans les liquides métalliques ont été obtenus avant la découverte des quasicristaux et l'étude de leur liquide d'équilibre et avant la mise au point récente de dispositifs expérimentaux permettant l'étude des liquides surfondus. citons néanmoins la présence d'un épaulement observé sur la droite de la seconde oscillation du facteur de structure d'alliages liquides de composition voisine de celle des amorphes métal-métaloïde $\mathrm{Fe}_{80} \mathrm{~B}_{20}[24,34]$ et métallique $\mathrm{Mgg}_{73} \mathrm{Zn}_{28}$ [23]. Dans ce dernier cas, la présence de l'épaulement est attribuée à de l'ordre local icosaédrique. En ce qui concerne les liquides simples, une expérience très sophistiquée utilisant le rayonnement synchrotron en incidence rasante a mis en évidence une symétrie d'ordre 5 dans la structure du plomb liquide à l'interface avec une surface de $\mathrm{Si}(001)$ [35]. ${ }^{4}$ Notons que la mise en évidence du caractère direc-

\footnotetext{
${ }^{4}$ Remarquons que cette expérience ne sonde pas le volume mais la structure de l'interface du liquide $\mathrm{Pb}$, qui peut être influencée par la symétrie locale du substrat Si.
} 


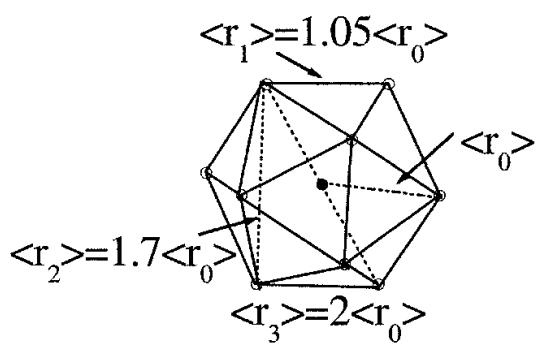

Figure 10 : agrégat icosaédrique.

tionnel des interactions dans les liquides métalliques basées sur un ordre local icosaédrique est une tâche très délicate car cet ordre local ne peut produire que des changements subtils des facteurs des structure mesurés. Pour illustrer cette problématique, la suite de ce cours est consacrée aux résultats des études concernant la structure des liquides métalliques monoatomiques surfondus d'une part et des alliages liquides en équilibre avec des phases icosaédriques d'autre part.

\section{Liquides surfondus}

Afin d'étudier la structure des liquides métalliques dans l'état surfondu, un dispositif à lévitation électromagnétique adapté aux expériences de diffusion de neutrons ou de RX a été développé [36]. Cette méthode produit une goutte de liquide sans interaction avec un porteéchantillon dans un environnement ultra-pur. Elle peut ainsi être surfondue de plusieurs centaines de degrés pendant une durée suffisante pour permettre l'obtention de spectres de très bonne qualité. Des expériences ont ainsi été réalisées sur plusieurs liquides monoatomiques tels que le Co [37] en diffusion de $\mathrm{RX}$ ou le $\mathrm{Ni}$, Fe et $\mathrm{Zr}$ [38] en diffusion de neutrons sur le spectromètre D20 à l'ILL (Grenoble).

Sur la figure 11 sont présentés les facteurs de structure, obtenus après les corrections standards, pour le Ni liquide à l'équilibre thermodynamique au dessus de la température de fusion $\mathrm{T}_{F}=1726 \mathrm{~K}$ et dans l'état surfondu (jusqu'à $17 \%$ de surfusion : $\mathrm{T}_{L}-290 \mathrm{~K}$ ). On observe très clairement un épaulement sur la droite de la seconde oscillation du facteur de structure qui s'accentue lorsque la température diminue et que l'on passe dans le régime surfondu. Ceci est également observé pour les autres liquides étudiés. Il est à noter que cet épaulement caractéristique est présent pour des liquides dont le produit de nucléation présente une structure aussi bien cfc pour le Ni que cc pour le Fe et le Zr.

Une analyse quantitative des fonctions de distributions de paires permet d'obtenir les distances interatomiques et les nombres de coordination. Alors que les distances premiers voisins n'évoluent pas significativement sur la gamme de température explorée, le nombre de coordination diminue légèrement quand la température augmente (passant de 11.2 à 12.3 pour le Ni entre 1905 et 1435 K). L'expansion thermique du liquide peut ainsi être interprétée comme un réarrangement de l'ordre local. Le nombre de coordination est globalement proche de 12 c'est-à-dire en accord avec un environnement atomique compact. Ceci n'est néanmoins pas suffisant pour discriminer entre différents environnements compacts tels que cfc, hc ou icosaédrique.

Pour obtenir des informations supplémentaires, une méthode de simulation des facteurs de 


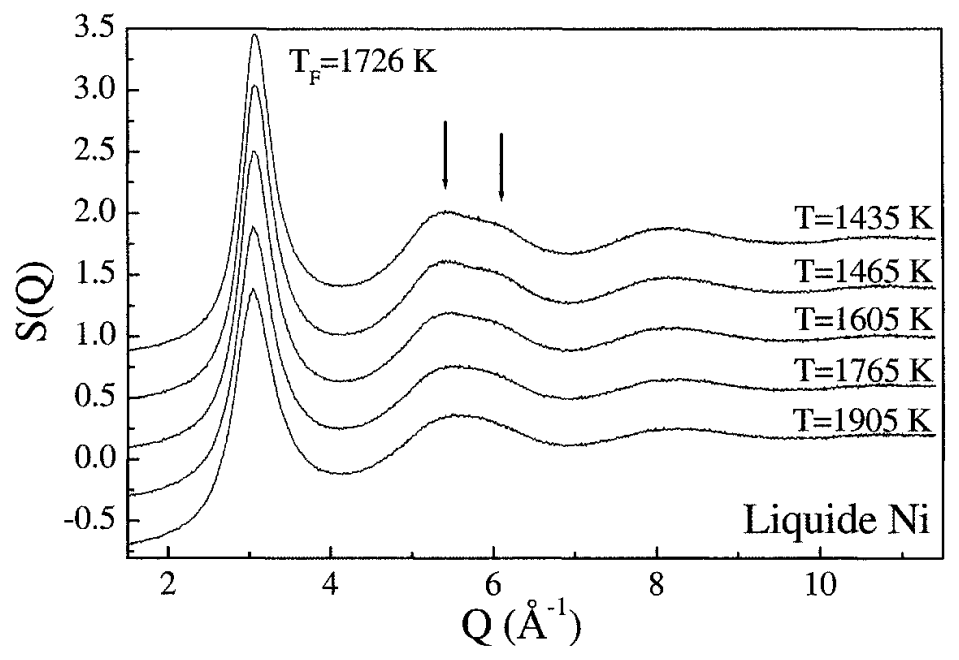

Figure 11 : Facteur de structure du Ni liquide à l'équilibre et dans l'état surfondu.

structure à grands vecteurs de diffusion a été employée. Ce type de simulation est simple avec peu de paramètres ajustables, qui plus est, directement liés aux données structurales. Cette approche a été initialement utilisée pour décrire des solides ou liquides moléculaires tels que le soufre [39] ou les cristaux de C60 [40]. La simulation du facteur de structure expérimental d'un liquide métallique est une tâche très difficile car les liaisons ne sont ni très fortes ni très directionnelles et l'aspect moléculaire n'est donc pas très marqué. Il est cependant envisageable de simuler la partie du facteur de structure à grands vecteurs de diffusion qui reflète essentiellement la contribution des atomes les plus proches sous certaines conditions. Le liquide doit pouvoir être décrit en termes d'agrégats c'est-à-dire être constitué d'entités dont les atomes sont plus rigidement liés les uns aux autres qu'avec le reste du liquide. On peut en effet toujours écrire le facteur de structure comme la somme des contributions de paires pondérées par un facteur de Debye-Waller provenant de l'agitation thermique. Ce facteur est d'autant plus petit à grands $Q$ que les atomes sont faiblement liés, comme c'est le cas pour les paires éloignées ou les paires d'atomes interagrégats. Ainsi, la contribution principale au facteur de structure à grands $Q$ provient des paires d'atomes intra-agrégat moyennant le fait que la durée de vie des agrégats $\tau_{c}$ est compatible avec le temps caractéristique de l'interaction neutronique $\left(\tau_{c}>10^{-10} \mathrm{~s}\right)$.

Pour établir le formalisme, partons de l'expression de la section efficace différentielle cohérente :

$$
\left(\frac{d \sigma}{d \Omega}\right)_{c o h} \propto \sum_{i j} b_{i} b_{j}<\exp \left\{i Q\left(R_{j}-R_{i}\right)\right\}>
$$

Avec $N$ le nombre total d'atomes dans l'alliage. Les contributions inter et intra-agrégat sont séparées en exprimant les positions atomiques $R_{j}$ en fonction de la position du centre de masse $R_{m}$ de l'agrégat, $R_{j}=R_{m}+r_{m j}$. La section efficace différentielle cohérente peut se réécrire :

$$
\left(\frac{d \sigma}{d \Omega}\right)_{c o h} \propto \sum_{m} \sum_{j(m)}^{n_{a}} \sum_{j^{\prime}(m)}^{n_{a}} b_{j} b_{j^{\prime}}<\exp \left\{i Q\left(r_{m j}-r_{m j^{\prime}}\right)\right\}>
$$




$$
+\sum_{m \neq m^{\prime}}<\exp \left\{i Q\left(R_{m}-R_{m^{\prime}}\right)\right\}>\sum_{j(m)}^{n_{a}} \sum_{j^{\prime}\left(m^{\prime}\right)}^{n_{a}} b_{j} b_{j^{\prime}}<\exp \left\{i Q\left(r_{m j}-r_{m^{\prime} j^{\prime}}\right)\right\}>
$$

Avec $n_{a}$ le nombre d'atomes dans les agrégats. On fait ensuite ressortir l'effet des fluctuations thermiques sur les positions atomiques autour de leur valeur moyenne que l'on sépare en mouvement translationnel du centre de masse $\exp (-2 W)$ et vibrations internes des agrégats $\exp \left(-2 W_{i j}\right)$ avec $2 W_{i j}=Q^{2}<\delta r_{i j}^{2}>/ 3$. A suffisamment grand $Q, \exp (-2 W)$ devient nul et le second terme de l'équation (12) disparaît. Pour une assemblée d'agrégats d'orientation aléatoire, en faisant la moyenne spatiale, on obtient finalement à grands $Q$ :

$$
S(Q)=\frac{1}{<b^{2}>}\left(\frac{d \sigma}{d \Omega}\right)_{c o h}=1+\frac{N_{m}}{N<b^{2}>} \sum_{i, j(i \neq j)}^{n_{a}} b_{i} b_{j} \frac{\sin \left(Q<r_{i j}>\right)}{Q<r_{i j}>} \exp \left(-2 w_{i j}\right)
$$

Avec $\left\langle r_{i j}>\right.$ la valeur moyenne de la distance entre les atomes $i$ et $j$ de l'agrégat $m, N_{m}$ le nombre d'agrégats. Si $N=n_{a} N_{m}$ tous les atomes sont dans les agrégats.

Les paramètres ajustables du modèle, une fois choisies la géométrie et la constitution chimique de l'agrégat, sont les distances inter-atomiques $r_{i j}$ d'une paire d'atomes $i$ et $j$ de l'agrégat, les fluctuations de ces distances $\left\langle\delta r_{i j}^{2}>\right.$ et la fraction des atomes du liquide compris dans les agrégats. Le nombre de paramètres est encore réduit par la relation entre les distances intraagrégat. Ainsi, on a pour le cas de l'icosaèdre, $r_{1}=1.0515 r_{0}, r_{2}=1.7013 r_{0}$ et $r_{3}=2 r_{0}$ (cf. Fig. 10). Notons également que les écarts quadratiques moyens $\left\langle\delta r_{i j}^{2}\right\rangle$ ont l'ordre de grandeur que laisse prévoir une variation linéaire en température à partir des valeurs des solides et que l'ajustement des simulations sur les facteurs de structure mesurés conduit à une évolution à peu près quadratique de ces facteurs $\left\langle\delta r_{i j}^{2}\right\rangle$ avec la distance inter-atomique.

Les résultats des simulations du facteur de structure du Ni liquide surfondu, obtenus avec cette méthode, sont présentés sur la figure 12. Une simulation basée sur un agrégat de type icosaédrique décrit mieux le spectre expérimental que celles basées sur des agrégats $\mathrm{cc}$, cfc ou hc, en particulier au niveau de la seconde oscillation du facteur de structure (autour de $5.5 \AA^{-1}$ ). Ceci peut qualitativement s'expliquer par la présence de deux courtes distances dans l'icosaèdre, qui produisent un élargissement, voire un épaulement, de la seconde oscillation du facteur de structure (cf. Fig. 12). Les distances inter-atomiques résultant de la simulation sont compatibles avec celles obtenues à partir des $g(r)$. Afin de déterminer la nature de l'ordre sur des distances un peu plus grandes, des simulations basées sur des agrégats constitués d'un plus grand nombre d'atomes ont été entreprises. Il en résulte qu'une simulation basée sur un agrégat de symétrie icosaédrique de 33 atomes (formé en ajoutant une couche dodécaèdrique sur l'icosaèdre initial) est en meilleur accord avec le facteur de structure mesuré qu'une simulation basée sur l'icosaèdre seul. Enfin, l'évolution des facteurs de structure mesurés quand la température augmente est bien reproduite lorsque le nombre total d'atomes du liquide compris dans les agrégats diminue et que le facteur de Debye-Waller augmente.

En résumé, la mesure de facteurs de structure de grande qualité dans l'état surfondu hors d'équilibre pour divers liquides métalliques monoatomiques a permis de mettre en évidence pour la première fois un épaulement sur la seconde oscillation du facteur de structure. Cette caractéristique n'est pas compatible avec un modèle de sphères dures mais est en accord avec un ordre local de type icosaédrique. En outre, cet ordre local est identique pour différentes structures des phases solides d'équilibre et semble s'intensifier lorsque la température diminue en particulier dans l'état surfondu. Quelque 50 ans après, l'ensemble de ces résultats permet de vérifier la prédiction de Frank [31]. 


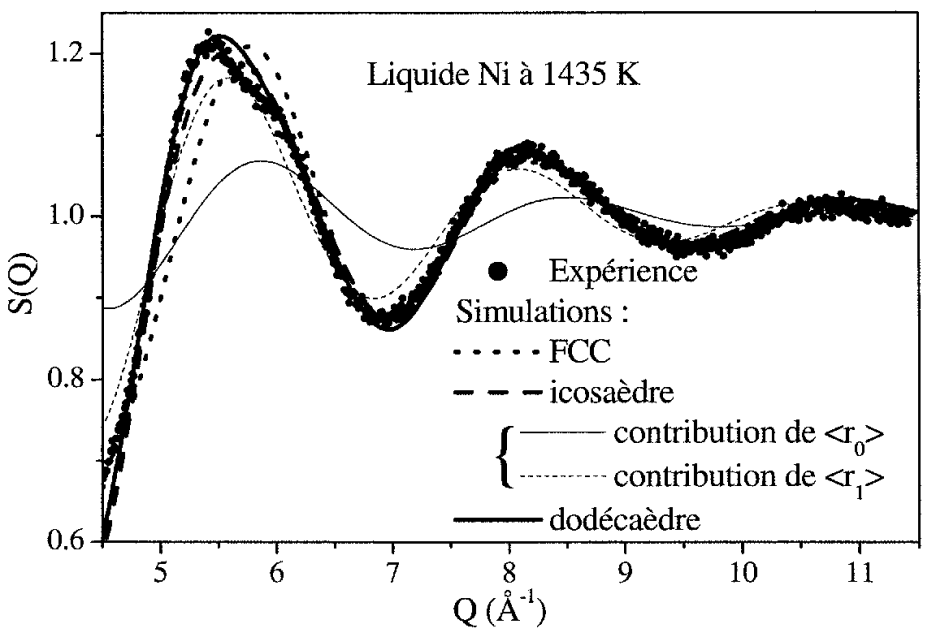

Figure 12 : Comparaison du facteur de structure mesuré du Ni liquide surfondu à $\mathrm{T}_{F}-290 \mathrm{~K}$ avec plusieurs simulations.

\section{Liquides en équilibre avec des quasicristaux}

La découverte des quasicristaux dans des alliages métalliques à base d'Al par Schechtman et al en 1984 [41] a initié une série d'études sur les liquides de composition voisine. Rappelons que les quasicristaux sont des phases ordonnées sans périodicité de translation mais avec un ordre orientationnel à grande distance. Ces phases présentent un ordre icosaédrique intrinsèque. Il existe des phases cristallines parentes des phases quasicristallines, appelées phases approximantes, qui possèdent un grand paramètre de maille et un ordre local identique à celui des phases quasicristallines correspondantes. L'ordre local icosaédrique est donc également présent dans ces phases approximantes ainsi que dans un grand nombre de phases intermétalliques des mêmes diagrammes de phase. C'est le cas des systèmes Al-Pd- $\mathrm{Mn}, \mathrm{Al}-\mathrm{Cr}$ ou Al-Mn riches en Al. On peut donc raisonnablement espérer que les liquides en équilibre avec ces phases solides pésenteront aussi un ordre local icosaédrique.

Dans la suite, nous exposerons différentes pistes d'analyse des facteurs de structure qui ont été employées pour mieux comprendre l'ordre chimique et topologique dans ces alliages liquides : comparaison des facteurs de structure des liquides et des phases solides en équilibre avec eux; détermination des facteurs de structure et fonctions de distribution de paires partiels; simulations des facteurs de structure à grands vecteurs de diffusion. Nous nous intéresserons aussi à l'évolution de l'ordre local en fonction de la température et à son incidence sur les propriétés magnétiques inhabituelles de ces liquides.

\subsection{Facteur de structure total}

La majorité des expériences qui sont présentées dans la suite a été réalisée sur les spectromètres 2-axes 7C2 au LLB (Saclay) et D4 à l'ILL (Grenoble) avec une longueur d'onde d'environ 0.7 A. Les échantillons, sour forme de cylindre, ont été placés dans un porte-échantillon en saphir et chauffés dans un four en vanadium ou en tungstène. Le porte-échantillon vide a été orienté autour de son axe vertical afin de minimiser la détection de pics de Bragg du saphir polycristallin. Le spectre de l'échantillon a été obtenu après correction de l'efficacité des cellules du détecteur et 


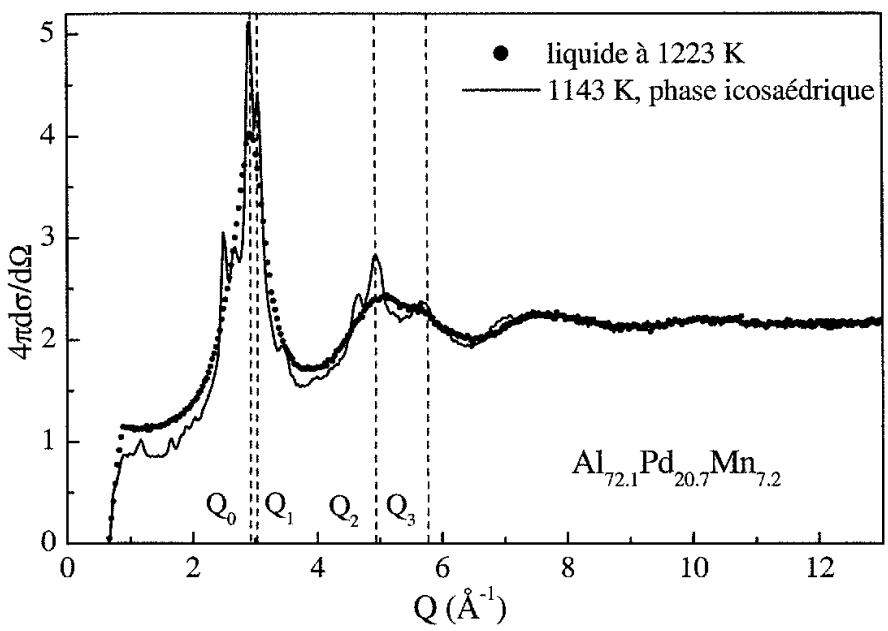

Figure 13 : Comparaison des sections efficaces différentielles de l'alliage $A 1_{72.1} \mathrm{Pd}_{20.7} \mathrm{Mn}_{7.2}$ dans l'état liquide et à une température où seule la phase icosaédrique est encore solide. Les droites verticales indiquent les positions des pics caractéristiques de l'ordre icosaédrique selon la théorie de Sachdev et Nelson $[30]$.

soustraction des contributions de l'environnement suivant la méthode de Paalman et Pings [42] qui permet également de corriger de l'auto-absorption de l'échantillon. Les spectres ont ensuite été corrigés des termes de diffusion inélastique [2] et de diffusion multiple [43] avant d'être normalisés en barns grace à une calibration avec un barreau de vanadium.

Tirer l'information structurale complète de l'analyse du facteur de structure d'un alliage ternaire est à priori une tâche très difficile. Certaines informations peuvent cependant être obtenues à partir du facteur de structure total comme nous allons l'illustrer dans le cas d'un liquide d'Al ${ }_{72.1} \mathrm{Pd}_{20.7} \mathrm{Mn}_{7.2}[44,45]$. La fusion de ce composé n'est pas congruente c'est-à-dire que les phases solides obtenues en cristallisation primaire n'ont pas la même composition que le liquide. La connaissance des diagrammes de phases a permis de choisir la composition de ce liquide de façon à ce qu'il forme de la phase icosaédrique parfaite en cristallisation primaire. Le liquide forme ultérieurement des phases approximantes et une phase riche en $\mathrm{Al}$.

L'allure générale de la section efficace différentielle mesurée à des températures supérieures à la température pour laquelle tout l'échantillon est liquide $\left(\mathrm{T}_{F}=1160 \mathrm{~K}\right)$ est présentée sur la figure 13. Notons tout d'abord le signal important à petits $Q$ avant la première oscillation du facteur de structure qui est dû à une diffusion paramagnétique de l'échantillon sur laquelle nous reviendrons dans la section 3.4 .

On remarque également, sur la droite de la deuxième oscillation, la présence d'un épaulement semblable à celui observé dans les amorphes métalliques et dans les liquides monoatomiques de la section précédente. La comparaison des spectres du liquide et du composé partiellement fondu, c'est-à-dire à une température où seule la phase icosaédrique est encore solide et donc à l'origine des pics de Bragg résiduels, donne des information sur l'origine de cette caractéristique. On observe que les groupes de pics de Bragg intenses de la phase icosaédrique correspondent précisément à des oscillations du facteur de structure du liquide avec en particulier deux groupes de pics de Bragg à l'emplacement des deux maxima de la seconde oscillation du liquide. Cette similitude suggère un ordre local proche dans les deux états.

D'après la théorie Sachdev et Nelson [30] mentionnée précédement, la symétrie icosaédrique 


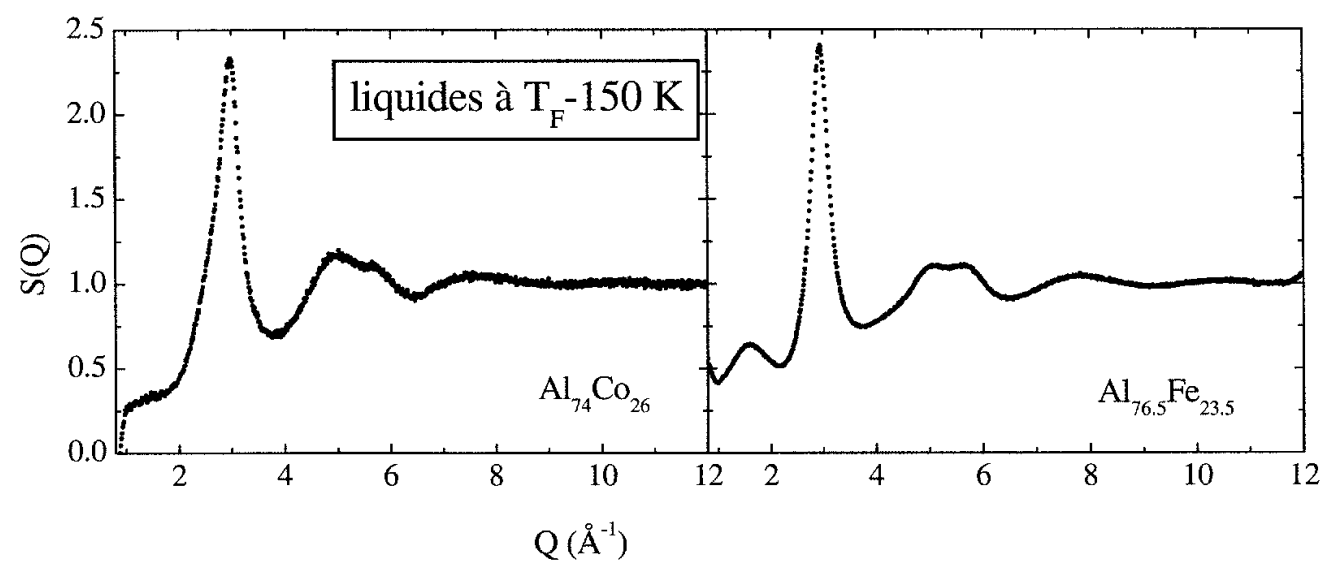

Figure 14 : Facteur de structure total des liquides surfondus $\mathrm{Al}_{74} \mathrm{Co}_{26}$ et $\mathrm{Al}_{13} \mathrm{Fe}_{4}$.

induit des maxima dans le facteur de structure à des positions caractéristiques $Q_{A}, Q_{B}$ et $Q_{C}$, correspondants respectivement au premier pic, au maximum de la seconde oscillation et à son épaulement. Ces valeurs de $Q$ peuvent être reliées à 4 taches de Bragg intenses des phases icosaédriques $Q_{0}, Q_{1}, Q_{2}$ et $Q_{3}$. Ces positions obéissent aux relations suivantes: $Q_{1}=1.052 Q_{0}$, $Q_{2}=1.701 Q_{0}$ et $Q_{3}=2 Q_{0}$. Cette théorie est parfaitement appliquable au facteur de structure de l'alliage $\mathrm{Al}_{72,1} \mathrm{Pd}_{20,7} \mathrm{Mn}_{7,2}$. Les quatre pics de Bragg de la phase icosaédrique, parmi les plus intenses sont indiqués par des lignes verticales et correspondent au premier maximum (assez large pour inclure $Q_{0}$ et $Q_{1}$ ) du facteur de structure du liquide, à la seconde oscillation et à son épaulement (cf. Fig. 13). Cette analyse est en faveur d'un ordre local icosaédrique similaire dans le solide et le liquide correspondant.

La présence d'une double structure sur la seconde oscillation du facteur de structure, qu'elle prenne la forme d'un épaulement, d'un dédoublement ou d'un sommet aplati en fonction du pouvoir diffusant respectif des différentes espéces chimiques, est générale dans les alliages à base d'Al formant des phases icosaédriques ou approximantes. C'est en effet le cas des liquides AI-Mn formant des phases icosaédriques métastables ou approximantes (cf. section suivante), Al-Cu-Co en équilibre avec des phases quasicristallines décagonales (quasipériodique à 2 dimensions) ou encore des liquides $\mathrm{Al}_{74} \mathrm{Co}_{26}$ (forment une phase décagonale métastable) et $\mathrm{Al}_{13} \mathrm{Fe}_{4}$ (de composition identique à celle d'une phase monoclinique approximante) (cf. Fig. 14) [46]. Notons dans ce dernier liquide la présence d'un prépic révélateur d'un ordre chimique dans ces alliages. L'analyse quantitative de l'ordre local et en particulier de cet ordre chimique nécessite d'autres outils tels que l'extraction des fonctions de distribution de paires partielles.

\subsection{Fonctions de distribution de paires partielles}

La détermination des fonctions de distribution de paires partielles dans le liquide ternaire $\mathrm{Al}_{72.1} \mathrm{Pd}_{20.7} \mathrm{Mn}_{7.2}$ est difficile en l'absence de substitution isotopique possible de l'Al et du $\mathrm{Pd}$. II faut donc se tourner vers des liquides mieux adaptés aux études de diffusion neutronique et qui présenteraient les mêmes caractéristiques. Une série d'expériences de diffusion de neutrons a été réalisée sur 5 liquides $\mathrm{Al}_{88.5}\left(\mathrm{Mn}_{x} \mathrm{Cr}_{1-x}\right)_{11.5}$ de rapport $\mathrm{Mn} / \mathrm{Cr}$ variable afin d'extraire les facteurs de structure et les fonctions de distribution de paires partielles [47]. Ce système a été choisi pour plusieurs raisons. Du point de vue de la diffusion de neutrons, un contraste important est attendu car le $\mathrm{Cr}\left(\mathrm{b}_{c}=0.3635\right)$ et le $\mathrm{Mn}\left(\mathrm{b}_{c}=-0.373\right)$ ont des longueurs de diffusion cohérentes pratiquement 


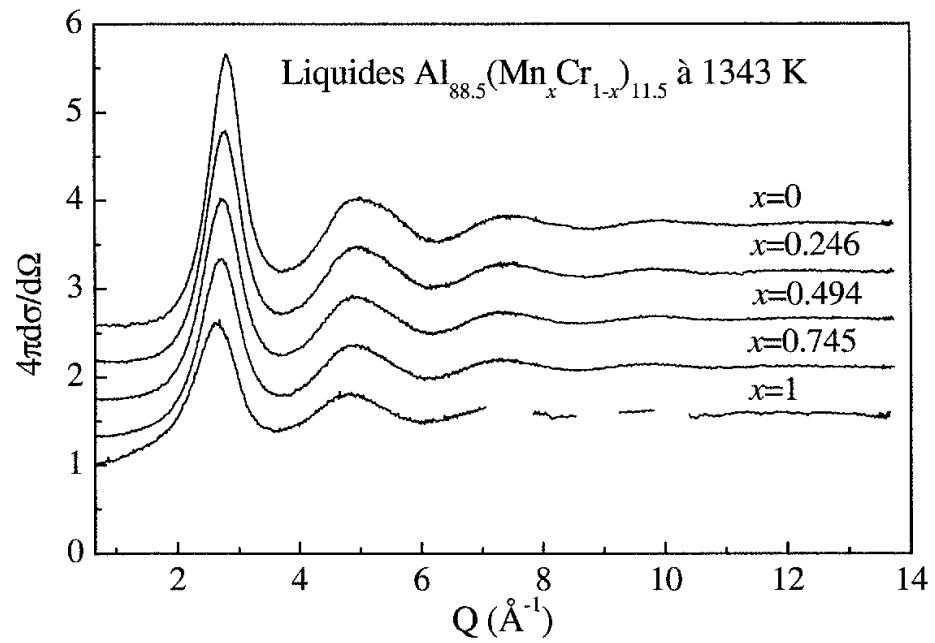

Figure 15 : Sections efficaces différentielles des 5 échantillons liquides $\mathrm{Al}_{88.5}\left(\mathrm{Mn}_{x} \mathrm{Cr}_{1-x}\right)_{11.5}$. Les spectres sont décalés de 0.25 barns.

opposées ${ }^{5}$. En outre, ces liquides sont en équilibre avec la phase $\mu-\mathrm{Al}_{4}\left(\mathrm{Mn}_{x} \mathrm{Cr}_{1-x}\right)$ [48], phase approximante de phases icosaédriques et qui présente une substitution isomorphe complète sur toute la gamme de rapport $\mathrm{Mn} / \mathrm{Cr}$ [49]. Ceci est une bonne indication d'une propriété similaire dans les liquides. Il faut cependant le vérifier car l'extraction des fonctions de distribution de paires partielles repose sur la propriété de substitution isomorphe. Celle-ci peut être testée de manière auto-cohérente après détermination des fonctions de distribution de paires partielles à partir d'un système surdimensionné.

Les sections efficaces différentielles résultantes sont présentées sur la figure $15^{6}$. L'amplitude des oscillations augmente et la position des maxima est décalée vers les grands $Q$ lorsque la concentration en Mn diminue. Ces évolutions sont dues uniquement à la différence entre les longueurs de diffusion $\mathrm{Mn} / \mathrm{Cr}$ comme cela sera explicité dans la suite. Les $S(Q)$ et $g(r)$ partiels de 5 liquides $\mathrm{Al}_{88.5}\left(\mathrm{Mn}_{x} \mathrm{Cr}_{1-x}\right)_{11.5}$ ont été extraits dans les deux formalismes de Bhatia-Thornton et Faber-Ziman. La validité de la substitution isomorphe a été testée via l'auto-cohérence de la procédure en vérifiant que les facteurs de structure partiels extraits à partir de toutes les combinaisons linéaires de 3 facteurs de structure mesurés sont identiques. Les partiels ont été finalement déterminés à partir des 4 spectres $x=0$ à 0.745 en utilisant une méthode adaptée au traitement de systèmes surdimensionnés [50].

Soit $\mathrm{M}=\mathrm{Cr}_{1-x} \mathrm{Mn}_{x}$, l'atome métal de transition moyen de l'alliage, les facteurs de structure partiels $\mathrm{S}_{A l A l}$ et $\mathrm{S}_{A l M}$ et $\mathrm{S}_{N N}$ et $\mathrm{S}_{C N}$ ainsi que les fonctions de distribution de paires correspondantes sont présentés sur la figure 16. Les partiels $S_{M M}$ et $S_{C C}$ n'ont pu être extraits avec une précision suffisante à cause de la proportion faible d'atomes de transition dans les liquides. Les positions du maximum des premiers pics $\mathrm{g}_{A l M}$ et $\mathrm{g}_{A l A l}$ sont respectivement 2.65 et $2.78 \AA$. On peut remarquer que le rapport de ces distances est très proche (1.049) du rapport attendu entre les deux premières distances d'un icosaèdre (1.0515). Les nombres de coordination ont été

\footnotetext{
${ }^{5} \mathrm{La}$ longueur de diffusion n'est pas continûment variable en fonction du numéro atomique dans le cas de la diffusion neutronique. Elle peut même être négative pour certains éléments, à l'inverse du facteur de diffusion des RX.

${ }^{6}$ Le spectre du liquide $\mathrm{Al}_{88.5} \mathrm{Mn}_{11.5}$ présentait des accidents à partir de $7 \AA^{-1}$, dus à des contaminations par des pics de Bragg du porte échantillon en saphir. Les points correspondants ont été supprimés.
} 


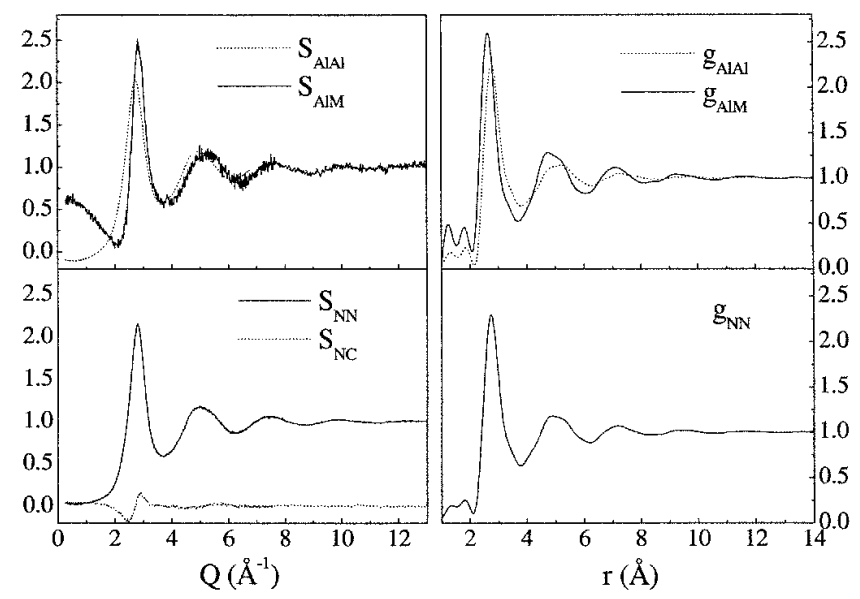

Figure 16 : Facteurs de structure et fonctions de distribution de paires partiels déterminés à partir des spectres des liquides $\mathrm{Al}_{88.5}\left(\mathrm{Mn}_{x} \mathrm{Cr}_{1-x}\right)_{11.5}$ d'après les formalismes de Faber-Ziman et Bhatia-Thornton.

obtenus par intégration de la première oscillation des RDF. Ils sont de 10.5 et 10.6 pour les paires $\mathrm{M}-\mathrm{Al}$ et Al-Al et de 11.9 pour le nombre de coordination total autour d'un atome. L'ensemble de ces données structurales est en faveur d'un ordre local icosaédrique. Une information sur l'ordre chimique peut également être obtenue. Ainsi, le premier pic de $\mathrm{g}_{A l M}$ est plus étroit et plus intense que celui de $\mathrm{g}_{A l A l}$. A grande distance interatomique, les oscillations de $\mathrm{g}_{A l M}$ présentent une amplitude plus importante que les oscillations de $\mathrm{g}_{A l A l}$. Les corrélations Al-M sont donc plus fortes que les corrélations Al-Al dans ces liquides ce qui montre l'existence d'un ordre chimique Al-M. Cet ordre chimique couplé à un rapport de taille atomique favorable (petit effet de taille visible sur le $\mathrm{S}_{N C}$ ) pourrait être à l'origine de l'ordre local icosaédrique dans les phases solides Al-M d'équilibre.

Les fonctions de distribution de paires partielles ont été précédemment déterminées par M. Maret et coll. dans un alliage $\mathrm{Al}_{80}\left(\mathrm{Mn}_{x}(\mathrm{FeCr})_{1-x}\right)_{20}$ de même composition qu'une phase icosaédrique métastable en utilisant la nature isomorphe de la substitution du Mn par le mélange équiatomique $\mathrm{Fe}-\mathrm{Cr}$ [51]. Ces auteurs se sont essentiellement intéressés à la nature de l'ordre topologique. Leur analyse se base en particulier sur la forme du partiel NN, à savoir la hauteur du premier pic par rapport aux oscillation suivantes et la position de l'épaulement sur la seconde oscillation compatible avec la description de l'ordre local icosaédrique de Sachdev et Nelson [30]. Pour comparaison, les fonctions de distribution de paires partielles ont également été déterminées dans l'alliage liquide $\mathrm{Al}_{80} \mathrm{Ni}_{20}$, qui ne forme pas de phase icosaédrique, en utilisant la substitution isotopique du $\mathrm{Ni}$ [52]. L'ordre topologique icosaédrique dans ce liquide semble moins marqué. Les mêmes auteurs ont mené une étude en fonction de la concentration en déterminant les fonctions $\mathrm{S}_{N N}$ et $\mathrm{g}_{N N}$ dans des liquides $\mathrm{Al}-\mathrm{M}$ avec différentes concentrations $(14,20,26.7,40 \%)$ en atomes métal de transition $\mathrm{M}=\mathrm{Mn}_{0.304}(\mathrm{FeCr})_{0.696}$. Ceci a permis de démontrer que l'ordre topologique peut être décrit par un ordre local icosaédrique quelle que soit la concentration [53]. Enfin, les fonctions de distribution de paires partielles du liquide $\mathrm{Al}_{71} \mathrm{Pd}_{19}\left(\mathrm{Mn}_{x}(\mathrm{FeCr})_{1-x}\right)_{10}$, qui n'ont pu être que partiellement déterminées, suggèrent à nouveau un ordre local icosaédrique [54]. Des analyses complémentaires basées sur des méthodes de simulations ont été entreprises afin de confirmer la nature de l'ordre local dans ce type de liquides. 


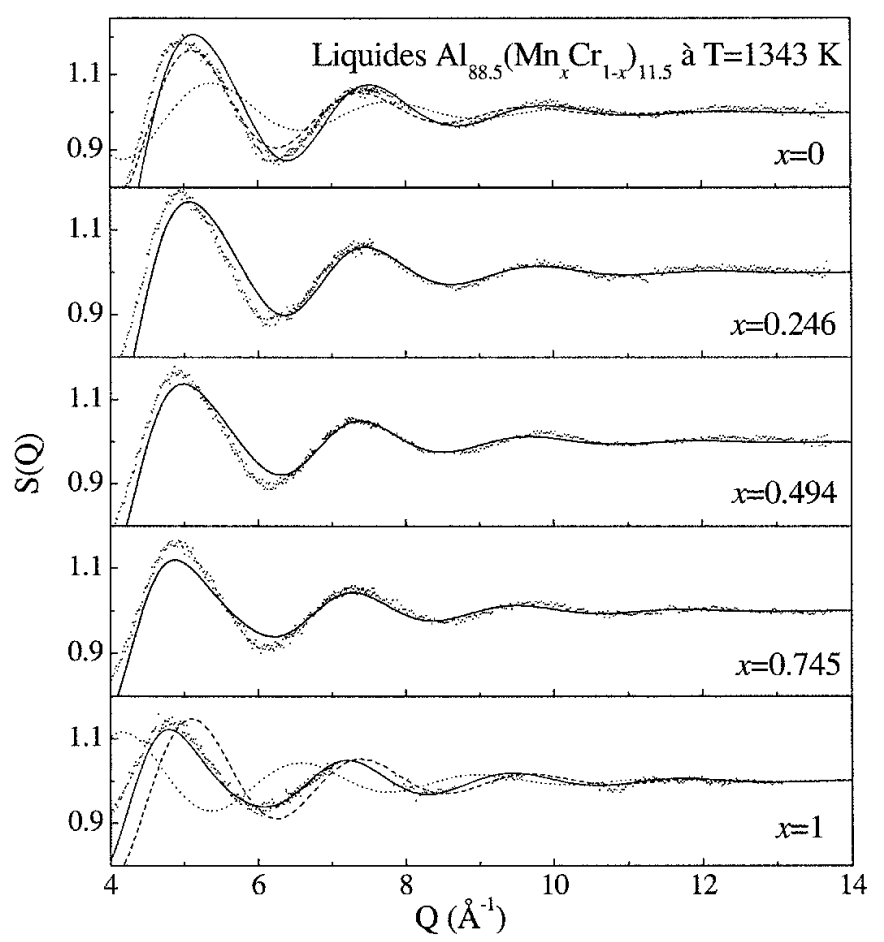

Figure 17 : Comparaison pour $Q>4 \AA^{-1}$ des facteurs de structure mesurés (points) des liquides $\mathrm{Al}_{88.5}\left(\mathrm{Mn}_{x} \mathrm{Cr}_{1-x}\right)_{11.5}$ et calculés (ligne solide) à partir d'un d'agrégat icosaédrique (AlM) ${ }_{12} \mathrm{M}$. Pour les deux alliages binaires, les contributions des paires d'atomes à des distances de $\left\langle r_{0}\right\rangle$ (ligne en pointillée) et $\left\langle r_{1}\right\rangle$ (ligne en tiret) sont tracées.

\subsection{Simulations des facteurs de structure}

M. Maret et coll. ont étudié les symétries locales d'assemblées d'atomes générées par dynamique moléculaire à partir des potentiels d'interaction inter-atomique extraits des fonctions de distribution de paires partielles mesurées et/ou de méthodes de Monte Carlo Inverse [55,56]. L'ordre local a été analysé en terme de polyèdres de Voronoï, c'est-à-dire construits autour de chaque atome à partir des plans bissecteurs des segments de droite joignant l'atome central à ses plus proches voisins. $2.2 \%$ des polyèdres obtenus au maximum ont une symétrie icosaédrique dans le liquide $\mathrm{Al}_{80}\left(\mathrm{Mn}_{x}(\mathrm{CrFe})_{1-x}\right)_{20}$ ce qui correspond à $22 \%$ des atomes du liquide participant à l'ordre local icosaédrique. Cette proportion est environ deux fois plus faible que dans le quasicristal mais est 10 fois plus grande que dans l'alliage $\mathrm{Al}_{80} \mathrm{Ni}_{20}$.

Les méthodes numériques utilisées par M. Maret et coll. sont très puissantes mais assez lourdes de mise en œuvre. L'alternative présentée ici est la méthode de simulation simple des facteurs de structure à grands vecteurs de diffusion décrite dans la section précédente. Cette méthode a tout d'abord été appliquée aux liquides $\mathrm{Al}_{88.5}\left(\mathrm{Mn}_{x} \mathrm{Cr}_{1-x}\right)_{11.5}$ [47] en s'appuyant sur les résultats des fonctions de distribution de paires partielles. Le facteur de structure d'un icosaèdre centré sur un atome de transition et dont la composition en $\mathrm{Al}$ et $\mathrm{M}$ correspond à la stoechiométrie de l'alliage 


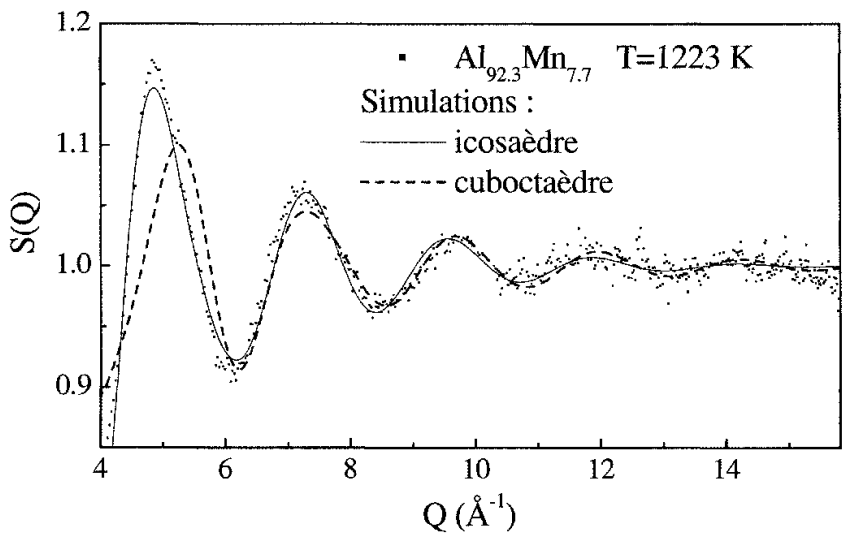

Figure 18 : Comparaison pour $Q>4 \AA^{-1}$ du facteur de structure mesuré (points) du liquide $\mathrm{Al}_{12} \mathrm{Mn}$ et calculé à partir d'un d'agrégat $\mathrm{Al}_{12} \mathrm{M}$ icosaédrique (ligne solide) et cuboctaédrique (ligne pointillée).

résiduel a ainsi été comparé aux facteurs de structure mesurés pour les différents rapports $\mathrm{Mn} / \mathrm{Cr}$. Tous les atomes du liquide simulé sont compris dans les agrégats et la proportion d'atomes de transition situés au centre d'un icosaèdre est de $67 \%$. Le résultat est reporté sur la figure 17 pour les 5 liquides. Les simulations sont en bon accord avec les facteurs de structure mesurés pour $Q>6.5 \AA^{-1}$. Pour des valeurs de $Q$ plus petites, les contributions inter-agrégats deviennent nonnégligeables. Les valeurs des paramètres $\left\langle r_{i j}\right\rangle$ et $\left\langle\delta r_{i j}\right\rangle$ obtenues sont les mêmes pour les 5 alliages. Les variations observées dans l'amplitude et la position des oscillations simulées sont donc uniquement dues à la variation de la longueur de diffusion moyenne de l'atome de transition. En particulier, le changement de forme de la seconde oscillation avec le rapport $\mathrm{Cr} / \mathrm{Mn}$ est dû au déplacement des oscillations de la contribution $\left\langle r_{0}\right\rangle$ (concerne essentiellement les courtes distances $\mathrm{M}-\mathrm{Al}$ ) vers les petites vecteurs de diffusion alors que la contribution $\left\langle r_{1}\right\rangle$ change très peu (essentiellement courtes distances $\mathrm{Al}-\mathrm{Al}$ ). L'influence respective des deux contributions est illustré pour les deux liquides binaires sur la figure 17. Enfin, notons que le bon accord entre expérience et simulation entérine la nature isomorphe de la substitution $\mathrm{Cr} / \mathrm{Mn}$.

L'intérêt de cette méthode de simulation est qu'elle peut également être appliquée à des liquides même lorsqu'on ne dispose pas des trois facteurs de structure nécessaires à la détermination des $S(Q)$ et $g(r)$ partiels. Cette méthode a ainsi été utilisée pour rendre compte du facteur de structure mesuré d'un liquide $\mathrm{Al}_{92.3} \mathrm{Mn}_{7.7}$ [47]. Ce liquide est, tout comme le liquide $\mathrm{Al}_{88.5} \mathrm{Mn}_{11.5}$ précédemment étudié, en équilibre avec la phase $\mu-\mathrm{Al}_{4} \mathrm{Mn}$. Sa concentration plus faible en $\mathrm{Mn}$ permet idéalement d'accommoder tous les Mn du liquide au centre d'icosaèdres d'Al. Ce modèle permet d'obtenir une très bonne simulation du facteur de structure mesuré pour des valeurs de $Q$ supérieures à $4 \AA^{-1}$ avec une distance $\left\langle r_{0}\right\rangle$ de $2.59 \AA$ alors qu'une simulation basée sur d'autres types d'agrégats tels que le cuboctaèdre (correspondant à la structure cfc) donne un bien moins bon accord (cf. Fig. 18). La même méthode de simulation appliquée a des liquides ternaires Al-Pd-Mn a montré que l'ordre local de ces derniers fait intervenir deux types d'agrégats icosaédriques, principalement formés d'Al et centrés sur des atomes de $\mathrm{Mn}$ et des atomes de $\mathrm{Pd}$ [47]. 


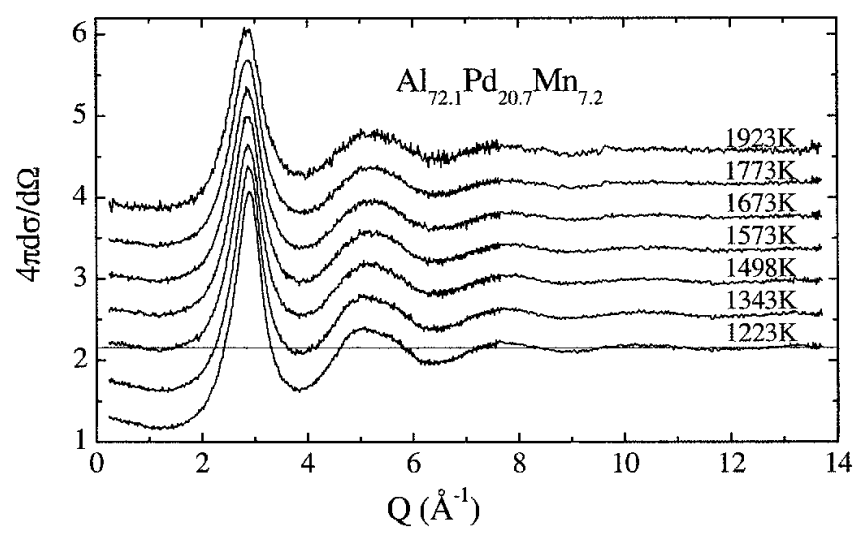

Figure 19 : Evolution en fonction de la température de la section efficace différentielle du liquide $\mathrm{Al}_{72.1} \mathrm{Pd}_{20.7} \mathrm{Mn}_{7.2}$. Les courbes aux différentes températures sont decalées successivement de 0.5 barns.

\subsection{Lien avec les propriétés physiques}

Dans les sections précédentes, nous avons présenté une analyse des facteurs de structure de plusieurs liquides à base d'Al et d'éléments de transition, en équilibre avec des phases ayant un ordre local icosaédrique. La détermination des fonctions de distribution de paires partielles et la simulation des facteurs de structure à grands $Q$ à partir de modèles d'agrégats, ont permis de prouver la présence d'un ordre local de type icosaédrique dans tous ces liquides. Cet ordre local semble caractérisé par un ordre chimique, les atomes de transition $(\mathrm{Mn}, \mathrm{Cr}, \mathrm{Pd})$ s'entourant préférentiellement d'un icosaèdre principalement constitué d'Al. Ces études ont été menées sur des facteurs de structure mesurés à une température proche de la température de fusion. On peut à présent s'interroger sur l'évolution de cet ordre local en fonction de la température et sur son incidence sur les propriétés physiques de ces liquides.

Le facteur de structure du liquide $\mathrm{Al}_{72.1} \mathrm{Pd}_{20.7} \mathrm{Mn}_{7.2}$ a été mesuré sur une large gamme de température, depuis $\mathrm{T}_{F}$ jusqu'à presque $2000 \mathrm{~K}$. On observe des changements du facteur de structure quand la température augmente (cf. Fig. 19) à savoir une diminution de l'amplitude du premier pic, un amortissement des oscillations successives, et la disparition progressive de l'épaulement de la seconde oscillation du facteur de structure clairement visible lorsque la température augmente. Ceci pourrait être lié à la destruction progressive de l'ordre local icosaédrique avec l'augmentation de la température mais l'augmentation de l'agitation thermique et donc du facteur de Debye-Waller peut aussi contribuer à cet effet.

Un autre phénomène remarquable affecte le facteur de structure, à savoir l'évolution en fonction de la température du signal à petits $Q:$ on observe une augmentation importante de ce signal à la fusion et une augmentation dans le liquide avec la température. Cette évolution est reportée sur la figure 20 pour le liquide $\mathrm{Al}_{72.1} \mathrm{Pd}_{20.7} \mathrm{Mn}_{7.2}$ à $Q=0.2 \AA^{-1}$. Rappelons que les neutrons, possédant un spin, peuvent interagir avec le moment des atomes magnétiques dans l'échantillon. On peut écrire la section efficace différentielle magnétique comme suit :

$$
\left(\frac{d^{2} \sigma}{d \Omega d E^{\prime}}\right)_{m a g}=\frac{\left(\gamma r_{0}\right)^{2}}{\pi} \frac{k^{\prime}}{k}|F(Q)|^{2}\left[\frac{\omega}{1-\exp \left(-\omega / k_{B} T\right)}\right] \frac{\chi(Q)}{g^{2} \mu_{B}^{2}} f(Q, \omega)
$$

avec $\gamma$ le facteur gyromagnétique, $r_{0}=\mu_{0} e^{2} / 4 \pi m_{e}=2.81810^{-1.5} \mathrm{~m}, g$ le facteur de Landé, $F(Q)$ le facteur de forme magnétique, $\chi(Q)$ la susceptibilité statique, $f(Q, \omega)$ décrivant la dynamique 


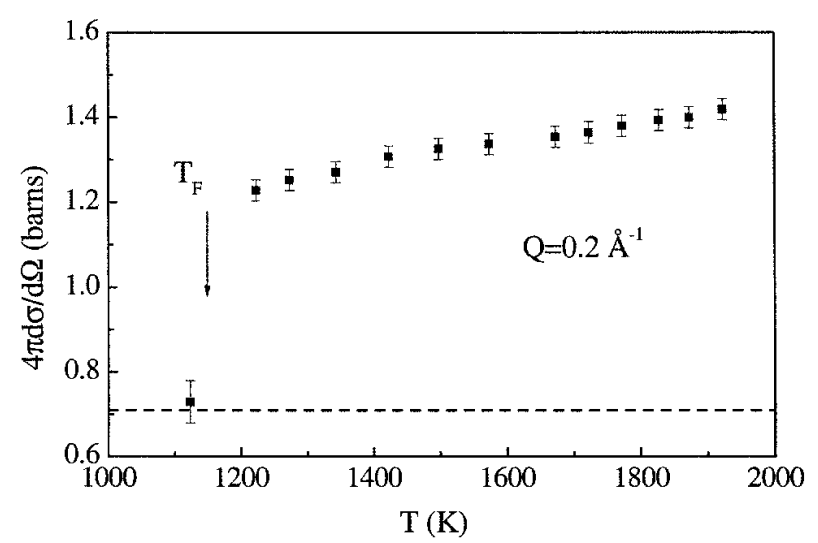

Figure 20 : Dépendance en température de la diffusion paramagnétique de neutrons à $Q=0.2 \AA^{-1}$. La ligne pointillée indique le maximum des contributions nucléaires.

des spins $\left(\int f(Q, \omega) d \omega=1\right)$.

En l'absence d'interactions entre les spins, $\chi(Q)$ et $f(Q, \omega)$ sont indépendants de $Q$. Si $c$ est la concentration en atomes magnétiques et $S$ le spin des atomes magnétiques, on a, après intégration sur l'énergie $\omega$ :

$$
\begin{gathered}
\chi(Q)=c g^{2} \mu_{B}^{2} \frac{S(S+1)}{3 k_{B} T} \\
\left(\frac{d \sigma}{d \Omega}\right)_{m a g}=\frac{2}{3} c \gamma^{2} r_{0}^{2}\left(\frac{1}{2} g F(Q)\right)^{2} S(S+1)
\end{gathered}
$$

Dans le cas où il y a des interactions entre moments magnétiques $(S)$ via les électrons de conduction (de spin $s$ ) par l'Hamiltonien d'échange de Heisenberg $-J \vec{S} . \vec{s}$, le terme $f(Q, \omega)$ prend la forme:

$$
f(Q, \omega)=\frac{1}{\pi} \frac{\delta}{\Delta^{2}+\omega^{2}}
$$

où $\Delta=\pi k_{B} T J^{2} n\left(E_{F}\right)^{2}$ avec $n\left(E_{F}\right)$ la densité d'états électroniques au niveau de Fermi par direction de spin.

L'origine magnétique du signal à petit $Q$ mesuré dans le liquide $\mathrm{Al}_{72.1} \mathrm{Pd}_{20.7} \mathrm{Mn}_{7.2}$ a été confirmée par des mesures du carré du facteur de forme magnétique sur le spectromètre D7 à l'ILL en utilisant des neutrons polarisés avec analyse de polarisation $[44,45]$. Cette méthode permet de séparer les contributions nucléaires et magnétiques. On obtient bien un signal magnétique nul dans le solide et un signal magnétique dans le liquide dû aux atomes de Mn (cf. Fig. 21). On peut noter que l'intensité de ce signal, une fois calibrée en barns, est très inférieure au signal mesuré à petits $Q$ sur $7 \mathrm{C} 2$ ou $\mathrm{D} 4$, corrigé de la contribution nucléaire. Ce problème est lié à l'étroitesse de la fenêtre d'intégration en énergie accessible sur D7, comparée à celles des spectromètres de poudres $7 \mathrm{C} 2$ et $\mathrm{D} 4$, qui ne permet donc pas de mesurer tout le signal magnétique car celui-ci a une extension en énergie. Une mesure de diffusion inélastique effectuée sur un spectromètre temps de vol (IN6, ILL) a permis de démontrer la nature quasiélastique du signal magnétique dans ces liquides due à la dynamique des spins des électrons de conduction interagissant avec les moments magnétiques (cf. équations (14) et (17)). Le signal total quasiélastique (cf. Fig. 21) peut 

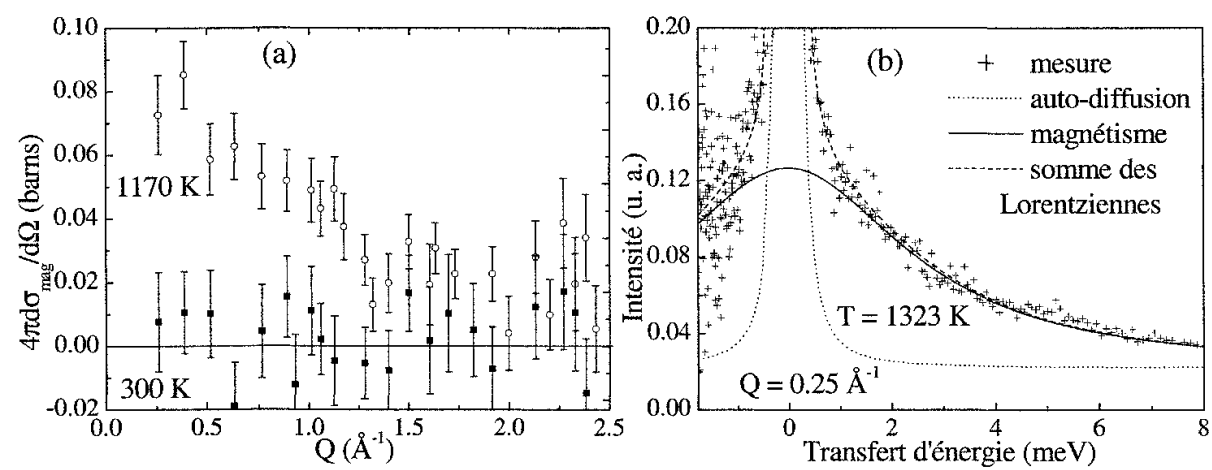

Figure 21 : (a) : Dédendance en $Q$ de la diffusion paramagnétique mesurée dans l'alliage $\mathrm{Al}_{72.1} \mathrm{Pd}_{20.7} \mathrm{Mn}_{7.2}$ solide et liquide en utilisant des neutrons polarisés. (b) : Signal quasiélastique mesuré dans le liquide $\mathrm{Al}_{72.1} \mathrm{Pd}_{20.7} \mathrm{Mn}_{7.2}$ par temps de vol sur IN6.

être décomposé en deux lorentziennes, dont l'une, étroite, est due à l'auto-diffusion des atomes et l'autre, plus large (d'environ $3 \mathrm{meV}$ de demi largeur à mi-hauteur), est attribuée à la diffusion magnétique. La largeur de cette dernière lorentzienne augmente avec le vecteur de diffusion et avec la température.

L'évolution en température du produit de la susceptibilité magnétique avec la température a la même allure que l'augmentation de la diffusion paramagnétique de neutrons. En utilisant une image d'alliages dilués [44,45], les valeurs de $c S(S+1)$, évaluées d'après les deux types de mesures sont compatibles (cf. équations (15) et (16)). Cette évolution est très surprenante puisque $\chi$ augmente avec $\mathrm{T}$ ce qui va à l'inverse d'une loi de Curie, généralement observée pour des liquides métalliques contenant des impuretés magnétiques (cas du CuMn pour comparaison [57]). L'augmentation du paramagnétisme avec $\mathrm{T}$ peut s'expliquer soit par une augmentation de la valeur du moment des atomes magnétiques, soit par l'augmentation du nombre de moments magnétiques dans le liquide. Soulignons que ce comportement est également observé dans les liquides $\mathrm{Al}-\mathrm{Mn}$ ou $\mathrm{Al}-\mathrm{Mn}-\mathrm{Cr}$.

L'interprétation de ce comportement est probablement liée à l'évolution de l'ordre local dans le liquide. Trambly et coll. [58] ont effectué des calculs de structure électronique dans diverses phases solides du système Al-Mn afin d'en comprendre les propriétés magnétiques et en particulier les très faibles valeurs, voire l'absence de magnétisme. Dans ces structures quasipériodiques ou approximantes, les auteurs ont mis en évidence le rôle du potentiel de paires $\mathrm{Mn}-\mathrm{Mn}$, qui est fort et oscillant de type Friedel avec une amplitude dépendant de l'état magnétique des atomes de Mn. La minimisation de l'énergie du système implique que les atomes de $\mathrm{Mn}$ se trouvent à des distances particulières non favorables au développement d'un moment magnétique. Dans le liquide, ce potentiel de paire est amorti mais peut subsister à courte portée s'il y a un ordre local réminiscent de celui du solide. Ceci pourrait expliquer le fait que tous les atomes de Mn ne sont pas magnétiques dans ces liquides juste au dessus du point de fusion mais le deviennent progressivement lorsque la température augmente et que l'ordre local icosaédrique se détériore.

Pour tester ce scénario, il serait essentiel d'avoir accès à l'évolution en fonction de la température de l'ordre local autour des atomes de transition (responsables du magnétisme) par la détermination de la fonction de distribution de paires $g_{M M}$. Une piste intéressante pour cette investigation est la technique de l'EXAFS (Extended X ray Absorption Fine Structure) qui, grâce à sa sélectivité 
chimique obtenue par le choix du seuil d'absorption, permettrait de sonder directement l'environnement local autour des atomes de transition. Notons cependant que l'EXAFS, appliqué à des systèmes désordonnés contenant plusieurs espèces chimiques à haute température, pose un certain nombre de problèmes d'ordre expérimental et d'analyse (importance de l'agitation thermique, nécessité d'aller au delà de l'approximation harmonique pour décrire les distributions atomiques radiales en tenant compte du désordre important). En conséquence, les expériences d'EXAFS ne sont souvent pas bien adaptées pour déterminer la structure local d'un liquide métallique à haute température. En revanche, cette technique est très sensible aux évolutions de l'ordre local en fonction d'un paramètre externe tel que la température ou la pression. L'EXAFS est donc un bon complément d'expériences de diffraction. La faisabilité de ce type.d'experiences a été demontrée par des mesures d'EXAFS au seuil du Co, réalisées par Egry et coll, sur un liquide métallique $\mathrm{Al}_{65} \mathrm{Cu}_{25} \mathrm{Co}_{10}$ en équilibre avec des quasicristaux [59].

\section{Conclusion}

Le modèle de sphères dures où l'interaction inter-atomique est isotrope et de nature répulsive a permis d'obtenir une description raisonnable, au premier ordre, de la structure des liquides métalliques, reproduisant les caractéristiques principales des facteurs de structures mesurés en diffraction de RX ou de neutrons. Néanmoins, il semble nécessaire de raffiner ce modèle simple pour prendre en compte la partie attractive et la nature plus ou moins directionnelle des interactions inter-atomiques. En effet, c'est de l'écart au comportement de sphères dures que dépendent toutes les propriétés originales des liquides métalliques, telles que l'ordre chimique, l'évolution en température, les transitions électroniques (par exemple métal-non métal), les propriétés magnétiques etc. Cette démarche a été illustrée avec l'exemple récent de l'étude de l'ordre local icosaédrique. Ce type d'ordre local semble en effet être un bon candidat pour décrire la nature légèrement directionnelle des interactions dans certains liquides : dans les liquides monoatomiques où il augmente quand la température diminue et en particulier dans l'état surfondu, dans les liquides $\mathrm{Al}-(\mathrm{Pd})-\mathrm{Mn}$ en équilibre avec des quasicristaux où il est basé sur un ordre chimique $\mathrm{Al}$-atome métal de transition. Ceci pourrait être lié au mécanisme de formation et de stabilisation des phases à symétrie icosaédrique dans ces systèmes. Le comportement magnétique surprenant de ces liquides est probablement également lié à l'évolution de l'ordre local icosaédrique avec la température. Cette recherche devrait se poursuivre avec le développement d'autres modèles et de techniques d'analyse plus sensibles afin de mettre en évidence de nouveaux comportements dans les liquides métalliques.

\section{Remerciements}

Les auteurs remercient tous leurs collaborateurs dans ce domaine de recherche: T. Schenk, D. Holland-Moritz, D. Herlach du DLR (Cologne, Allemagne), et F. Hippert, M. Audier du LMGP (ENSPG, Saint Martin d'Hères, France). 


\section{Références}

1. P. Debye and H. Menke, Physik. Z. 31, 797 (1930).

2. G. Placzek, Phys. Rev. 86, 377 (1952).

3. T. Iida, R. I. L. Guthrie, The physical properties of Liquid Metals, (Clarendon Press Oxford, 1988).

4. J. M. Ziman, Philos. Mag. 6, 1013 (1961).

5. Y. Waseda, the structure of non-crystalline materials, (McGraw-Hill International Book company, 1980).

6. Y. Waseda and S. Tamaki, Phil. Mag. 32, 273 (1975).

7. H. Ocken and C. N. J. Wagner, Phys. Rev 149, 122 (1966).

8. N. W. Ashcroft and D. C. Langreth, Phys. Rev. B 156, 685 (1967).

9. T.E. Faber et J.M. Ziman, Phil. Mag. 11, 153 (1965).

10. A.B. Bhatia et D.E. Thornton, Phys. Rev. B 2, 3004 (1970).

11. M-C. Bellissent-Funel, P-J. Desre, Philos. Mag. 36, 1063 (1977).

12. J. Blétry, Z. Naturf. a 31, 960 (1976).

13. J. E. Enderby, D. M. North, and P. A. Egelstaff., Phil. Mag. 14, 961 (1966).

14. Y. Waseda, J. Phys. F, 7, L151 (1977).

15. J. Blétry, Z. Naturforsch. 33a, 327 (1977).

16. J. Blétry, and J.-F. Sadoc, J. Phys. F 5, L110 (1975).

17. H. Reiter, H. Ruppersberg, and W. Speicher, 3rd Int. Conf. on Liquid Metals, 133 (Bristol 1976).

18. M. Favre Bonte, J. Blétry, P. Hicter et al., J. Phys. Colloque, 41, C8-156 (1980).

19. P. Lamparter, W. Martin, S. Steeb et al., Z. Naturforsch. 33a, 329 (1983).

20. H. T. J. Reijers, M.-L. Sabouji, D. Price et al., Phys. Rev. B 40, 6018 (1989).

21. W. van der Lugt, J. Phys. Condens. Matter 8, 6115 (1996).

22. P. K. Leung and J. G. Wright, Philos. mag. 30, 185 (1974).

23. P. Andonov and P. Chieux, J. Non-Cryst. Sol. 93, 331 (1987).

24. E. Nold, P. Lamparter, H. Olbrich. et al., Z. Naturf. a 36, 1032 (1981).

25. J. D. Bernal, Proc. Roy. Soc. A280, 299 (1964).

26. C. H. Bennett, J. Appl. Phys. 43, 2727 (1972).

27. T. Ichikawa, Physica Status Solidi A 29, 293 (1975).

28. J. Blétry, Z. Naturf. a 32, 445 (1977).

29. J. F. Sadoc, J. Dixmier and A. Guinier, J. Non-Cryst. Sol. 46, 12 (1973).

30. S. Sachdev and D. R. Nelson, Phys. Rev. Lett. 53, 1947 (1984).

31. F. C. Frank, Proc. R. Soc. London A 215, 43 (1952).

32. D. Turnbull, Undercooled Alloy Phases, (E. W. Collings and C. C. Koch, The Metallurgical Society, Warrendale 1987).

33. D. Holland-Moritz, Int. J. Non-Equilib. Process. 11, 169 (1998).

34. E. Nold, G. Rainer-Harbach, P. Lamparter et al., Z. Naturf. a 38, 325 (1983). 
35. H. Reichert, O. Klein, H. Dosch et al., Nature 408, 839 (2000).

36. C. Notthoff, H. Franz, M. Hanfland, D. M. Herlach, D. Holland-Moritz, et W. Petry, Rev. Sci. Instrum. 713791 (2000).

37. D. Holland-Moritz, T. Schenk, R. Bellissent, V. Simonet, K. Funakoshi, J. Merino Alvarez, T. Buslaps, and S. Reutzel, à paratre dans J. of Non-Cryst. Solids (2002).

38. T. Schenk, D. Holland-Moritz, V. Simonet, R. Bellissent, and D. M. Herlach, Phys. Rev. Lett. 8975507 (2002).

39. R. Bellissent, L. Descotes and P. Pfeuty, J. Phys. Condens. Matter 6, A211 (1994).

40. P. Damay, F. Leclercq and P. Chieux, Phys. Rev. B 41, 9676 (1990); F. Leclercq, P. Damay, M. Foukani et al. Phys. Rev. B 48, 2748 (1993).

41. D. Schechtman, I. Blech, D. Gratias et al., Phys. Rev. Lett. 53, 1951 (1984).

42. H. H. Paalman and C. J. Pings, J. Appl. Phys. 33, 2635 (1962).

43. I. A. Blech and B. L. Averbach, Phys. Rev. 137, A1113 (1965).

44. F. Hippert, M. Audier, H. Klein et al., Phys. Rev. Lett. 76, 54 (1996).

45. V. Simonet, F. Hippert, H. Klein et al., Phys. Rev. B 58, 5273 (1998).

46. D. Holland-Moritz, T. Schenk, V. Simonet et al., à l'impression dans J. Alloys and Compounds (2002).

47. V. Simonet, F. Hippert, M. Audier et al., Phys. Rev. B 65, 24203 (2001).

48. C. Brink-Shoemaker, D. A. Keszler, D. P. Shoemaker, Acta Crystallogr. B 45, 13 (1989).

49. T. Schenk, H. Klein, M. Audier et al., Philos. Mag. Lett. 76, 189 (1997).

50. J. P. Simon, O. Lyon and D. de Fontaine, J. Appl. Cryst. 18, 230 (1985).

51. M. Maret, A. Pasturel, C. Senillou et al., J. Phys. (France) 50, 295 (1989).

52. M. Maret, T. Pomme, A. Pasturel et al., Phys. Rev. B 42, 1598 (1990).

53. M. Maret, P. Chieux, J. M. Dubois et al., J. Phys. Condens. Matter 3, 2801 (1991).

54. M. Maret, J. M. Dubois and P. Chieux, J. Non-Cryst. Solids 156-158, 918 (1993).

55. M. Maret, F. Lançon and L. Billard, J. Phys. I France 3, 1873 (1993).

56. M. Maret, F. Lançon and L. Billard, J. Phys. Condens. Matter 6, 5791 (1994).

57. O. F. Gruber and J. A. Gardner, Phys. Rev. B 4, 3994 (1971).

58. G. Trambly de Laissardière, D. Mayou, V. Simonet, et al., J. of Mag. \& Mag. Mat 226, 1029 (2001).

59. D. Holland-Moritz, G. Jacobs and I. Egry, Mat. Sci. Eng. A 294-296, 369 (2000). 